\title{
On the Existence of a Classical Optimal Solution and of an Almost Strongly Optimal Solution for an Infinite-Horizon Control Problem
}

\author{
Dominika Bogusz
}

Received: 6 June 2011 / Accepted: 13 July 2012 / Published online: 1 August 2012

(C) The Author(s) 2012. This article is published with open access at Springerlink.com

\begin{abstract}
We consider an infinite-horizon optimal control problem with the cost functional described either by an integral over an unbounded interval (a Lebesgue integral) or by a limit of integrals (an improper Lebesgue integral). We prove some theorems on the existence of solutions to such problems. The proofs are based on appropriate lower closure theorems and some extensions of Olech's theorem on the lower semicontinuity of an integral functional; these extensions cover the cases of functionals described by an integral over an unbounded interval and by a limit of integrals.
\end{abstract}

Keywords Infinite-horizon optimal control - Existence of an optimal solution · Lower closure theorem · Olech's theorem

\section{Introduction}

This paper is devoted to an infinite-horizon optimal control problem described by an ordinary differential equation. The cost functional is given either by an integral over an unbounded interval (a Lebesgue integral) or by a limit of integrals (an improper Lebesgue integral). The next section provides a simple example that illustrates the difference between the two functionals.

The problem corresponds to many phenomena that are of interest, for instance, in management science or economics: It can model the relationship between advertising, sales, and company profit or describe some production-inventory systems; see [1].

Communicated by Lars Grüne.

D. Bogusz (凶)

Department of Econometrics, Faculty of Economics and Sociology, University of Łódź, Łódź, Poland e-mail: bodomi@math.uni.lodz.pl 
The first difficulty we encounter when analyzing this problem is how to define appropriately an optimal pair. The literature offers many different definitions; see, for example, [2]. We introduce two new concepts of an optimal pair: a classical optimal pair for the model with an integral over an unbounded interval and an almost strongly optimal pair for the model with a limit of integrals (an improper Lebesgue integral). Compared to known definitions, these new concepts are a more natural extension of the definition of an optimal pair for finite-horizon models. Some relation between known and new definitions is shown in Sect. 2.

Having adequately defined an optimal pair, we give some conditions that ensure the existence of an optimal pair in the class of locally absolutely continuous trajectories and measurable controls. Here we use the method presented in [3]. It is based on the concept of the modified Lagrangian, and on a suitable version of the lower closure theorem for multifunctions defined over an unbounded domain.

The lower closure theorem, for a bounded domain, can be found in [4, Theorem 10.7.i]. Some variants of this theorem have been obtained in [5] for a special form of the multifunction and in [6] where the assumptions involve some "equi-behavior" of integrals over a bounded interval. We prove some versions of this theoremTheorem 6.1 and Theorem 6.2-for multifunctions in a general form, defined on the interval $[0, \infty[$. Such a theorem, with slightly different assumptions, has been stated in [3] without proof. The proofs of our lower closure theorems are based on some extensions of the classical Olech's theorem on the lower semicontinuity of an integral functional to the case of functions defined on an unbounded domain; see [7].

Our paper consists of seven main sections. In Sect. 2, we describe the model under study in detail and give an elementary example to justify this paper. Section 3 recalls some properties of locally absolutely continuous functions defined on the interval $[0, \infty[$. Section 4 is devoted to the classical Olech's theorem on the lower semicontinuity of an integral functional that involves an integral over a set of finite measure and some counterparts of this result for the functionals $\left(J_{f}\right)$ and $\left(J_{\text {lim }}\right)$ with integrands that depend on four variables and with an integral over the interval $[0, \infty[$. Section 5 concerns the concept of the modified Lagrangian and its basic properties. In Sect. 6, the lower closure theorems for the above-mentioned functionals are proven. In Sect. 7, theorems on the existence of an optimal solution to system (P) with the cost functional $\left(J_{f}\right)$ or $\left(J_{\text {lim }}\right)$ are derived and some examples that illustrate the existence theorems are given. In Sect. 8, some optimality principles are given. These principles say that an optimal solution of the infinite-horizon optimal control problem given by $(\mathrm{P})$ and $\left(J_{f}\right)$ or $(\mathrm{P})$ and $\left(J_{\lim }\right)$ is optimal on each finite time interval, in the usual sense.

\section{Motivation}

Consider the infinite-horizon control system

$$
\begin{cases}\dot{x}(t)=f(t, x(t), u(t)) & \text { for a.e. } t \in[0, \infty[, \\ x(0)=0, & \text { for } t \in[0, \infty[, \\ x(t) \in A(t) & \text { for a.e. } t \in[0, \infty[ \\ u(t) \in U(t, x(t)) & \end{cases}
$$


with the cost functional

$$
J_{f}(x, u)=\int_{0}^{\infty} F(t, x(t), u(t)) d t,
$$

where $f:\left[0, \infty\left[\times \mathbb{R}^{n} \times \mathbb{R}^{m} \rightarrow \mathbb{R}^{n}, F:\left[0, \infty\left[\times \mathbb{R}^{n} \times \mathbb{R}^{m} \rightarrow \mathbb{R}, A:\left[0, \infty\left[\rightrightarrows \mathbb{R}^{n 1}\right.\right.\right.\right.\right.\right.$, and $U: \mathrm{Gr} A \rightrightarrows \mathbb{R}^{m}$. The set $\mathrm{Gr} A$ is the graph of the multifunction $A$.

All integrals will hereafter signify Lebesgue integration.

A function $g:[0, \infty[\rightarrow \mathbb{R}$ is said to be summable iff the integrals of the positive part $g_{+}=\max \{g, 0\}$ and the negative part $g_{-}=\max \{-g, 0\}$ are finite; the function $g$ is said to be integrable iff at least one of these integrals is finite. The (proper) Lebesgue integral of $g$ is $\int_{0}^{\infty} g(t) d t:=\int_{0}^{\infty} g_{+}(t) d t-\int_{0}^{\infty} g_{-}(t) d t$ in both cases. See [8].

If a function $f:[0, \infty[\rightarrow \mathbb{R}$ is summable on each interval $] 0, T$ [ with $T$ positive, then the improper Lebesgue integral is defined to be the $\operatorname{limit}_{1} \lim _{T \rightarrow \infty} \int_{0}^{T} f(x) d x$ whenever it exists. See [9, Chap. VII, Sect. 8] or [10, Chap. VIII, Sect. 8].

Using the integral $\int_{0}^{\infty} F(t, x(t), u(t)) d t$ makes it necessary to impose some conditions that ensure the summability of the function $F(\cdot, x(\cdot), u(\cdot))$ on the unbounded interval $[0, \infty$ [. Such conditions are restrictive and not always satisfied in real-life applications; cf. Gale's cake eating problem in [3] and [4]. It, therefore, seems reasonable (necessary) to consider another notion of optimality. To weaken the assumptions on $F$, consider the functional

$$
J_{\lim }(x, u):=\lim _{T \rightarrow \infty} \int_{0}^{T} F(t, x(t), u(t)) d t
$$

instead of $J_{f}$. In such a case, it is enough to assume that $F(\cdot, x(\cdot), u(\cdot))$ is locally summable (that is, summable on each bounded subinterval of $[0, \infty[)$ and there exists a finite $\operatorname{limit}_{T \rightarrow \infty} \int_{0}^{T} F(t, x(t), u(t)) d t$.

The difference between $J_{\lim }(x, u)$ and $J_{\int}(x, u)$ can be better seen if one takes the function $h(t)=\frac{\sin t}{t}$ : the integral $\int_{0}^{\infty} h(t) d t$ does not exist, yet $\lim _{T \rightarrow \infty} \int_{0}^{T} h(t) d t$ exists and is equal to $\frac{\pi}{2}$. In other words, the function $h$ is neither summable nor integrable and despite that there the improper Lebesgue integral of this function exists.

To sum up, this paper assumes that there exists one of the integrals: Lebesgue or, at least, improper Lebesgue. For the sake of the reader's convenience, we use distinct notation for them: $\int_{0}^{\infty}$ for the Lebesgue integral and $\lim _{T \rightarrow \infty} \int_{0}^{T}$ for the improper Lebesgue integral.

The monograph [3] introduces strong optimality: A pair $\left(x^{*}, u^{*}\right)$ is called strongly optimal iff

$$
\lim _{T \rightarrow \infty} \int_{0}^{T} F(t, x(t), u(t)) d t>-\infty
$$

and the inequality

$$
\lim _{T \rightarrow \infty} \int_{0}^{T}\left(F\left(t, x^{*}(t), u^{*}(t)\right)-F(t, x(t), u(t))\right) d t \leq 0
$$

\footnotetext{
${ }^{1}$ We assume that all multifunctions in this paper have nonempty sets as values.
} 
holds true for each pair $(x, u)$ which satisfies system $(\mathrm{P})$ and is such that the function $F(\cdot, x(\cdot), u(\cdot))$ is locally summable on $[0, \infty[$. It is easy to observe that strong optimality and the notion of optimality based on the functional $\left(J_{\text {lim }}\right)$ (see Definition 7.8) are not equivalent. More precisely: Suppose that the pair $(x, u)$ satisfying equation (P) is optimal in the sense of Definition 7.8; only if we assume that the function $F(\cdot, x(\cdot), u(\cdot))$ is locally integrable on $[0, \infty[$ and the limit $\lim _{T \rightarrow \infty} \int_{0}^{T} F(t, x(t), u(t)) d t$ exists, is it meaningful to speak about the truth of the inequality in the above definition. We have therefore decided to say that the pair optimal in the sense of Definition 7.8 is almost strongly optimal.

To the author's knowledge, the definition of an optimal pair for problem (P) with the cost functional $\left(J_{f}\right)$ (i.e., a classical optimal solution) was not considered in the literature. Different interpretations of the integral $\int_{0}^{\infty} f(t, x(t), u(t)) d t$ either in the Lebesgue sense or in the Riemann sense have been discussed in [11].

\section{Locally Absolutely Continuous Functions}

This section recalls a definition and some properties of locally absolutely continuous functions defined on the interval $[0, \infty[$.

A function $x:[0, \infty[\rightarrow \mathbb{R}$ is called locally absolutely continuous on $[0, \infty[$ iff the function $\left.x\right|_{[0, T]}$ is absolutely continuous on $[0, T]$ for each $T>0$.

The space of all locally absolutely continuous functions on $[0, \infty[$ will be denoted by $A C_{\text {loc }}([0, \infty[, \mathbb{R})$. It follows from the integral representation of absolutely continuous functions on a bounded interval that $x$ belongs to the space $A C_{\text {loc }}([0, \infty[, \mathbb{R})$ if and only if there exists a function $l \in L_{\text {loc }}^{1}([0, \infty[, \mathbb{R})$ and $c \in \mathbb{R}$ such that

$$
x(t)=\int_{0}^{t} l(s) d s+c
$$

for $t \in\left[0, \infty\left[\right.\right.$, where $L_{\text {loc }}^{1}([0, \infty[, \mathbb{R})$ is the space of locally summable functions on $\left[0, \infty\right.$ [. Consequently, each function $x \in A C_{\text {loc }}([0, \infty[, \mathbb{R})$ has the derivative $\dot{x}(t)$ almost everywhere (a.e.) on $[0, \infty[$.

We shall consider $A C_{\text {loc }}([0, \infty[, \mathbb{R})$ endowed with the topology generated by the family of seminorms

$$
p_{q}(x) \equiv \int_{0}^{q}|\dot{x}(s)| d s+|x(0)|,
$$

where $x \in A C_{\text {loc }}\left(\left[0, \infty[, \mathbb{R})\right.\right.$ and $q \in \mathbb{Q}^{+}$(the positive rationals). A sequence $\left\{x_{k}\right\}_{k \in \mathbb{N}} \subset A C_{\text {loc }}\left(\left[0, \infty[, \mathbb{R})\right.\right.$ converges to $x \in A C_{\text {loc }}([0, \infty[, \mathbb{R})$ iff

$$
p_{q}\left(x_{k}-x\right) \longrightarrow 0, \quad \text { when } k \rightarrow \infty
$$

for each $q \in \mathbb{Q}^{+}$. See [12, Theorem 1.37].

We can prove, in an elementary way, 
Theorem 3.1 Let $\Phi^{*}$ be a continuous linear functional on $A C_{\mathrm{loc}}([0, \infty[, \mathbb{R})$. There exists a function $g \in L^{\infty}\left(\left[0, \infty[, \mathbb{R})\right.\right.$ and a constant $c \in \mathbb{R}$ such that $\left.g\right|_{T_{1}, \infty[} \equiv 0$ for some $T_{1}>0$ and

$$
\Phi^{*}(x)=\int_{0}^{\infty} g(s) \dot{x}(s) d s+c x(0)
$$

for $x \in A C_{\mathrm{loc}}\left(\left[0, \infty[, \mathbb{R})\right.\right.$. Conversely, any functional $\Phi^{*}$ given by (1) is linear and continuous on $A C_{\mathrm{loc}}([0, \infty[, \mathbb{R})$.

The following characterization of weak convergence in $A C_{\text {loc }}([0, \infty[, \mathbb{R})$ results from the above theorem.

Theorem 3.2 A sequence $\left\{x_{k}\right\}_{n \in \mathbb{N}}$ is weakly convergent to $x$ in $A C_{\text {loc }}([0, \infty[, \mathbb{R})$ if and only if the following two conditions are satisfied:

(i) the sequence $\left\{\left.\dot{x}_{k}\right|_{[0, T]}\right\}_{k \in \mathbb{N}}$ is weakly convergent to $\left.\dot{x}\right|_{[0, T]}$ in $L^{1}([0, T], \mathbb{R})$ for any $T>0$,

(ii) the sequence $\left\{x_{k}(0)\right\}_{k \in \mathbb{N}}$ is convergent to $x(0)$ in $\mathbb{R}$.

This theorem implies the following two results:

Theorem 3.3 If a sequence $\left\{x_{k}\right\}_{k \in \mathbb{N}}$ is weakly convergent to $x$ in $A C_{\mathrm{loc}}([0, \infty[, \mathbb{R})$, then

(i) the sequence $\left\{x_{k}(t)\right\}_{k \in \mathbb{N}}$ is convergent to $x(t)$ for any $t \in[0, \infty[$,

(ii) the sequence $\left\{x_{k}\right\}_{k \in \mathbb{N}}$ is convergent to $x$ in $L_{\text {loc }}^{1}([0, \infty[, \mathbb{R})$.

The following theorem has been proved in [3, Theorem 7.1, p. 158]:

Theorem 3.4 $A$ set $B \subset A C_{\mathrm{loc}}([0, \infty[, \mathbb{R})$ is relatively weakly sequentially compact iff

(i) the family $\left.C_{1}\right|_{T}=\left\{\left.\dot{x}\right|_{[0, T]}: x \in B\right\}$ is equiabsolutely summable ${ }^{2}$ on $[0, T]$ for any $T>0$,

(ii) the set $\{x(0): x \in B\}$ is bounded in $\mathbb{R}$.

\section{Lower Semicontinuity of an Integral Functional}

Consider the integral functional

$$
I(x, u)=\int_{0}^{T} G(t, x(t), u(t)) d t
$$

where $G:[0, T] \times \mathbb{R}^{n} \times \mathbb{R}^{m} \rightarrow \mathbb{R} \cup\{+\infty\}$.

\footnotetext{
${ }^{2}$ Let $E$ be a Lebesgue measurable and bounded subset of $\mathbb{R}$. A family of summable functions $\left\{f_{S}\right.$ : $E \rightarrow \mathbb{R} ; s \in S\}$, where $S$ is an arbitrary nonempty set of indices, is equiabsolutely summable on $E$ iff for any $\varepsilon>0$ there exists a $\delta>0$ such that $\int_{F}\left|f_{S}\right| \leq \varepsilon$ for any $s \in S$ and for any measurable set $F \subseteq E$ with $|F|<\delta$, where $|F|$ is the Lebesgue measure of $F$.
} 
The following theorem has been proved in [7].

\section{Theorem 4.1 If}

(i) the function $G$ is a normal integrand ${ }^{3}$ on $[0, T] \times\left(\mathbb{R}^{n} \times \mathbb{R}^{m}\right)$,

(ii) the function $G(t, x, \cdot)$ is convex on $\mathbb{R}^{m}$ for any $(t, x) \in[0, T] \times \mathbb{R}^{n}$,

(iii) there exist a constant $M \in \mathbb{R}$ and a summable function $\Psi:[0, T] \rightarrow \mathbb{R}$ such that

$$
G(t, x, u) \geq \Psi(t)-M(|x|+|u|)
$$

for any $(t, x, u) \in[0, T] \times \mathbb{R}^{n} \times \mathbb{R}^{m}$,

then

$$
\liminf _{k \rightarrow \infty} I\left(x_{k}, u_{k}\right) \geq I\left(x_{0}, u_{0}\right)
$$

provided that the sequence $\left\{x_{k}\right\}_{k \in \mathbb{N}}$ converges to $x_{0}$ in $L^{1}\left([0, T], \mathbb{R}^{n}\right)$ and the sequence $\left\{u_{k}\right\}_{k \in \mathbb{N}}$ converges weakly to $u_{0}$ in $L^{1}\left([0, T], \mathbb{R}^{m}\right)$.

\subsection{Case of a (Proper) Lebesgue Integral}

Consider the integral functional

$$
I_{f}(x, \xi, \lambda)=\int_{0}^{\infty} l(t, x(t), \xi(t), \lambda(t)) d t
$$

where $l:\left[0, \infty\left[\times \mathbb{R}^{n} \times \mathbb{R}^{m} \times \mathbb{R} \rightarrow \mathbb{R} \cup\{+\infty\}\right.\right.$.

\section{Theorem 4.2 If}

(i) the function l is a normal integrand on $\left[0, \infty\left[\times\left(\mathbb{R}^{n} \times \mathbb{R}^{m+1}\right)\right.\right.$,

(ii) the function $l(t, x, \cdot, \cdot)$ is convex on $\mathbb{R}^{m} \times \mathbb{R}$ for any $(t, x) \in\left[0, \infty\left[\times \mathbb{R}^{n}\right.\right.$,

(iii) there exist a constant $M \in \mathbb{R}$ and a summable function $\Psi:[0, \infty[\rightarrow \mathbb{R}$ such that

$$
l(t, x, \xi, \lambda) \geq \Psi(t)+M \lambda
$$

for $(t, x, \xi, \lambda) \in\left[0, \infty\left[\times \mathbb{R}^{n} \times \mathbb{R}^{m} \times \mathbb{R}\right.\right.$,

then

$$
\liminf _{k \rightarrow \infty} I_{\int}\left(x_{k}, \xi_{k}, \lambda_{k}\right) \geq I_{\int}\left(x_{0}, \xi_{0}, \lambda_{0}\right),
$$

provided that the sequence $\left\{x_{k}\right\}_{k \in \mathbb{N}}$ converges to $x_{0}$ in $L_{\text {loc }}^{1}\left(\left[0, \infty\left[, \mathbb{R}^{n}\right)\right.\right.$, the sequence $\left\{\xi_{k}\right\}_{k \in \mathbb{N}}$ converges weakly to $\xi_{0}$ in $L_{\text {loc }}^{1}\left(\left[0, \infty\left[, \mathbb{R}^{m}\right)\right.\right.$, the sequence $\left\{\lambda_{k}\right\}_{k \in \mathbb{N}} \subset$ $L^{1}\left(\left[0, \infty[, \mathbb{R})\right.\right.$ converges weakly to $\lambda_{0} \in L^{1}\left(\left[0, \infty[, \mathbb{R})\right.\right.$ in $L_{\text {loc }}^{1}([0, \infty[, \mathbb{R})$, and

$$
\liminf _{k \rightarrow \infty} \int_{0}^{\infty} M \lambda_{k}(t) d t \geq \int_{0}^{\infty} M \lambda_{0}(t) d t .
$$

\footnotetext{
${ }^{3}$ A function $f:[0, T] \times \mathbb{R}^{k} \rightarrow \mathbb{R} \cup\{ \pm \infty\}$ is a normal integrand iff it is $\mathcal{L}([0, T]) \times \mathcal{B}\left(\mathbb{R}^{k}\right)$-measurable and the function $f(t, \cdot)$ is lower semicontinuous on $\mathbb{R}^{k}$ for any $t \in[0, T]$. Here, $\mathcal{L}([0, T])$ is the family of Lebesgue measurable subsets of the interval $[0, T]$ and $\mathcal{B}\left(\mathbb{R}^{k}\right)$ is the family of Borel subsets of $\mathbb{R}^{k}$.
} 
Remark 4.1 If the functions $x:\left[0, \infty\left[\rightarrow \mathbb{R}^{n}, \xi:\left[0, \infty\left[\rightarrow \mathbb{R}^{m}\right.\right.\right.\right.$, and $\lambda:[0, \infty[\rightarrow \mathbb{R}$ are Lebesgue measurable and $l$ is a normal integrand on $\left[0, \infty\left[\times\left(\mathbb{R}^{n} \times \mathbb{R}^{m} \times \mathbb{R}\right)\right.\right.$, then the map $[0, \infty[\ni t \rightarrow l(t, x(t), \xi(t), \lambda(t)) \in \mathbb{R}$ is Lebesgue measurable. See [13, Corollary $2 \mathrm{~B}]$.

Proof of Theorem 4.2 Notice that

$$
\begin{aligned}
l\left(\cdot, x_{k}(\cdot), \xi_{k}(\cdot), \lambda_{k}(\cdot)\right)= & {\left[l\left(\cdot, x_{k}(\cdot), \xi_{k}(\cdot), \lambda_{k}(\cdot)\right)-\Psi(\cdot)-M \lambda_{k}(\cdot)\right] } \\
& +\left[\Psi(\cdot)+M \lambda_{k}(\cdot)\right] .
\end{aligned}
$$

The function $l\left(\cdot, x_{k}(\cdot), \xi_{k}(\cdot), \lambda_{k}(\cdot)\right)$ is, therefore, integrable on $[0, \infty[$ as the sum of an integrable function (a nonnegative measurable function-see assumption (iii)) and a summable one. Consequently, $I_{f}\left(x_{k}, \xi_{k}, \lambda_{k}\right)$ is well defined for $k=0,1, \ldots$

Step 1. $\Psi \equiv 0, M=0$.

The function $l\left(\cdot, x_{k}(\cdot), \xi_{k}(\cdot), \lambda_{k}(\cdot)\right)$ is nonnegative in this case so

$$
\int_{0}^{\infty} l\left(t, x_{k}(t), \xi_{k}(t), \lambda_{k}(t)\right) d t \geq \int_{0}^{T} l\left(t, x_{k}(t), \xi_{k}(t), \lambda_{k}(t)\right) d t
$$

for any $T>0$ and $k=1,2, \ldots$.

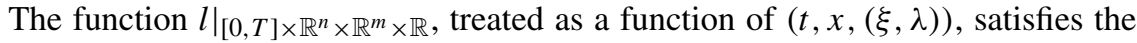
assumptions of Theorem 4.1. Moreover, the fact that the sequence $\left\{x_{k}\right\}_{k \in \mathbb{N}}$ converges to $x_{0}$ in $L_{\text {loc }}^{1}\left(\left[0, \infty\left[, \mathbb{R}^{n}\right)\right.\right.$ implies that the sequence $\left\{\left.x_{k}\right|_{[0, T]}\right\}$ converges to $\left.x_{0}\right|_{[0, T]}$ in $L^{1}\left([0, T], \mathbb{R}^{n}\right)$ for any $T>0$. The weak convergence of the sequence $\left\{\xi_{k}\right\}_{k \in \mathbb{N}}$ to $\xi_{0}$ in $L_{\text {loc }}^{1}\left(\left[0, \infty\left[, \mathbb{R}^{m}\right)\right.\right.$ and the weak convergence of the sequence $\left\{\lambda_{k}\right\}_{k \in \mathbb{N}}$ to $\lambda_{0}$ in $L_{\text {loc }}^{1}\left(\left[0, \infty[, \mathbb{R})\right.\right.$ imply the weak convergence of the sequence $\left\{\left(\xi_{k}, \lambda_{k}\right)\right\}_{k \in \mathbb{N}}$ to $\left(\xi_{0}, \lambda_{0}\right)$ in $L^{1}\left([0, T], \mathbb{R}^{m+1}\right)$ for any $T>0$. Hence, by Theorem 4.1,

$$
\liminf _{k \rightarrow \infty} \int_{0}^{T} l\left(t, x_{k}(t), \xi_{k}(t), \lambda_{k}(t)\right) d t \geq \int_{0}^{T} l\left(t, x_{0}(t), \xi_{0}(t), \lambda_{0}(t)\right) d t
$$

for any $T>0$.

It follows from (2) and (3) that

$$
\begin{aligned}
& \liminf _{k \rightarrow \infty} \int_{0}^{\infty} l\left(t, x_{k}(t), \xi_{k}(t), \lambda_{k}(t)\right) d t \\
& \quad=\liminf _{T \rightarrow \infty} \liminf _{k \rightarrow \infty} \int_{0}^{\infty} l\left(t, x_{k}(t), \xi_{k}(t), \lambda_{k}(t)\right) d t \\
& \quad \geq \liminf _{T \rightarrow \infty} \liminf _{k \rightarrow \infty} \int_{0}^{T} l\left(t, x_{k}(t), \xi_{k}(t), \lambda_{k}(t)\right) d t \\
& \quad \geq \liminf _{T \rightarrow \infty} \int_{0}^{T} l\left(t, x_{0}(t), \xi_{0}(t), \lambda_{0}(t)\right) d t \\
& \quad=\lim _{T \rightarrow \infty} \int_{0}^{T} l\left(t, x_{0}(t), \xi_{0}(t), \lambda_{0}(t)\right) d t=\int_{0}^{\infty} l\left(t, x_{0}(t), \xi_{0}(t), \lambda_{0}(t)\right) d t .
\end{aligned}
$$


The last equality results from the fact that $l$ is nonnegative. Hence, we get the assertion for $\Psi \equiv 0$ and $M=0$.

Step 2. The general case.

Consider the map

$$
\mathcal{A}(t, x, \xi, \lambda)=l(t, x, \xi, \lambda)-\Phi(t, x, \xi, \lambda)-\Upsilon(t, x, \xi, \lambda)
$$

where

$$
\Phi(t, x, \xi, \lambda)=\Psi(t) \text { and } \Upsilon(t, x, \xi, \lambda)=M \lambda
$$

The fact that $\Psi$ is summable on $[0, \infty[$ implies that the map $(t, x, \xi, \lambda) \rightarrow \Psi(t)$ is $\mathcal{L}\left(\left[0, \infty[) \times \mathcal{B}\left(\mathbb{R}^{n} \times \mathbb{R}^{m} \times \mathbb{R}\right)\right.\right.$-measurable. It can be inferred from the continuity of the map $(t, x, \xi, \lambda) \rightarrow M \lambda$ that it is $\mathcal{L}\left(\left[0, \infty[) \times \mathcal{B}\left(\mathbb{R}^{n} \times \mathbb{R}^{m} \times \mathbb{R}\right)\right.\right.$-measurable. For this reason, the map $\mathcal{A}$ is $\mathcal{L}\left(\left[0, \infty[) \times \mathcal{B}\left(\mathbb{R}^{n} \times \mathbb{R}^{m} \times \mathbb{R}\right)\right.\right.$-measurable. Besides, $\mathcal{A}(t, \cdot, \cdot, \cdot)$ is lower semicontinuous as the sum of the lower semicontinuous map $l(t, \cdot, \cdot, \cdot)$, the constant map $\Phi(t, \cdot, \cdot, \cdot)$, and the continuous map $\Upsilon(t, \cdot, \cdot, \cdot)$. The map $\mathcal{A}(t, x, \cdot, \cdot)$ is convex as the sum of the convex function $l(t, x, \cdot, \cdot)$, the constant function $\Phi(t, x, \cdot, \cdot)$, and the linear map $\Upsilon(t, x, \cdot, \cdot)$. Further, $\mathcal{A}(t, x, \xi, \lambda) \geq 0$ for $(t, x, \xi, \lambda) \in\left[0, \infty\left[\times \mathbb{R}^{n} \times \mathbb{R}^{n} \times \mathbb{R}\right.\right.$. Using the result obtained in Step 1 , it can be deduced that

$$
\liminf _{k \rightarrow \infty} \int_{0}^{\infty} \mathcal{A}\left(t, x_{k}(t), \xi_{k}(t), \lambda_{k}(t)\right) d t \geq \int_{0}^{\infty} \mathcal{A}\left(t, x_{0}(t), \xi_{0}(t), \lambda_{0}(t)\right) d t
$$

As a result,

$$
\begin{aligned}
& \liminf _{k \rightarrow \infty} \int_{0}^{\infty} l\left(t, x_{k}(t), \xi_{k}(t), \lambda_{k}(t)\right) d t \\
& =\liminf _{k \rightarrow \infty} \int_{0}^{\infty}\left[\left(l\left(t, x_{k}(t), \xi_{k}(t), \lambda_{k}(t)\right)-\Psi(t)-M \lambda_{k}(t)\right)+\Psi(t)+M \lambda_{k}(t)\right] d t \\
& \geq \liminf _{k \rightarrow \infty} \int_{0}^{\infty}\left(l\left(t, x_{k}(t), \xi_{k}(t), \lambda_{k}(t)\right)-\Psi(t)-M \lambda_{k}(t)\right) d t+\int_{0}^{\infty} \Psi(t) d t \\
& \quad+\liminf _{k \rightarrow \infty} \int_{0}^{\infty} M \lambda_{k}(t) d t \\
& \geq \int_{0}^{\infty}\left(l\left(t, x_{0}(t), \xi_{0}(t), \lambda_{0}(t)\right)-\Psi(t)-M \lambda_{0}(t)\right) d t \\
& \quad+\int_{0}^{\infty} \Psi(t) d t+\int_{0}^{\infty} M \lambda_{0}(t) d t \\
& =\int_{0}^{\infty} l\left(t, x_{0}(t), \xi_{0}(t), \lambda_{0}(t)\right) d t .
\end{aligned}
$$

The proof is over. 
4.2 Case of an Improper Lebesgue Integral

Now consider the functional

$$
I_{\lim }(x, \xi, \lambda)=\lim _{T \rightarrow \infty} \int_{0}^{T} l(t, x(t), \xi(t), \lambda(t)) d t
$$

where $l:\left[0, \infty\left[\times \mathbb{R}^{n} \times \mathbb{R}^{m} \times \mathbb{R} \rightarrow \mathbb{R} \cup\{+\infty\}\right.\right.$.

\section{Theorem 4.3 If}

(i) the function l is a normal integrand on $\left[0, \infty\left[\times\left(\mathbb{R}^{n} \times \mathbb{R}^{m+1}\right)\right.\right.$,

(ii) the function $l(t, x, \cdot, \cdot)$ is convex on $\mathbb{R}^{m} \times \mathbb{R}$ for any $(t, x) \in\left[0, \infty\left[\times \mathbb{R}^{n}\right.\right.$,

(iii) there exist a constant $M \in \mathbb{R}$ and a locally summable function $\Psi:[0, \infty[\rightarrow \mathbb{R}$ that satisfy $\lim _{T \rightarrow \infty} \int_{0}^{T} \Psi(t) d t>-\infty$ and $l(t, x, \xi, \lambda) \geq \Psi(t)+M \lambda$ for any $(t, x, \xi, \lambda) \in\left[0, \infty\left[\times \mathbb{R}^{n} \times \mathbb{R}^{m} \times \mathbb{R}\right.\right.$,

then

$$
\liminf _{k \rightarrow \infty} I_{\lim }\left(x_{k}, \xi_{k}, \lambda_{k}\right) \geq I_{\lim }\left(x_{0}, \xi_{0}, \lambda_{0}\right),
$$

provided that the sequence $\left\{x_{k}\right\}_{k \in \mathbb{N}}$ converges to $x_{0}$ in $L_{\text {loc }}^{1}\left(\left[0, \infty\left[, \mathbb{R}^{n}\right)\right.\right.$; the sequence $\left\{\xi_{k}\right\}_{k \in \mathbb{N}}$ converges weakly to $\xi_{0}$ in $L_{\mathrm{loc}}^{1}\left(\left[0, \infty\left[, \mathbb{R}^{m}\right) ; \lambda_{k} \in L_{\mathrm{loc}}^{1}([0, \infty[, \mathbb{R}) ;\right.\right.$ there exists a limit $\lim _{T \rightarrow \infty} \int_{0}^{T} M \lambda_{k}(t) d t>-\infty$ for $k=0,1, \ldots$; the sequence $\left\{\lambda_{k}\right\}_{k \in \mathbb{N}}$ converges weakly to $\lambda_{0}$ in $L_{\text {loc }}^{1}([0, \infty[, \mathbb{R})$; and

$$
\liminf _{k \rightarrow \infty} \lim _{T \rightarrow \infty} \int_{0}^{T} M \lambda_{k}(t) d t \geq \lim _{T \rightarrow \infty} \int_{0}^{T} M \lambda_{0}(t) d t .
$$

Proof It follows from the equality

$$
\begin{aligned}
l\left(\cdot, x_{k}(\cdot), \xi_{k}(\cdot), \lambda_{k}(\cdot)\right)= & {\left[l\left(\cdot, x_{k}(\cdot), \xi_{k}(\cdot), \lambda_{k}(\cdot)\right)-\Psi(\cdot)-M \lambda_{k}(\cdot)\right] } \\
& +\left[\Psi(\cdot)+M \lambda_{k}(\cdot)\right]
\end{aligned}
$$

that the function $l\left(\cdot, x_{k}(\cdot), \xi_{k}(\cdot), \lambda_{k}(\cdot)\right)$ is locally integrable on $[0, \infty[$ as the sum of an integrable function (a nonnegative measurable function) and a locally summable function. The existence of the limits

$$
\begin{aligned}
& \lim _{T \rightarrow \infty} \int_{0}^{T}\left(l\left(t, x_{k}(t), \xi_{k}(t), \lambda_{k}(t)\right)-\Psi(t)-M \lambda_{k}(t)\right) d t, \\
& \lim _{T \rightarrow \infty} \int_{0}^{T} \Psi(t) d t, \text { and } \lim _{T \rightarrow \infty} \int_{0}^{T} M \lambda_{k}(t) d t
\end{aligned}
$$

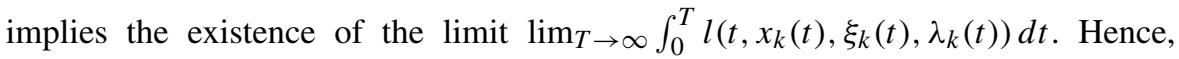
$I_{\lim }\left(x_{k}, \xi_{k}, \lambda_{k}\right)$ is well defined for $k=0,1, \ldots$ 
Step 1. $\Psi \equiv 0, M=0$.

The function $l\left(\cdot, x_{k}(\cdot), \xi_{k}(\cdot), \lambda_{k}(\cdot)\right)$ is nonnegative in this case so

$$
\lim _{T \rightarrow \infty} \int_{0}^{T} l\left(t, x_{k}(t), \xi_{k}(t), \lambda_{k}(t)\right) d t \geq \int_{0}^{S} l\left(t, x_{k}(t), \xi_{k}(t), \lambda_{k}(t)\right) d t
$$

for any $S>0$ and $k=1,2, \ldots$

Similarly as in the proof of Theorem 4.2,

$$
\liminf _{k \rightarrow \infty} \int_{0}^{T} l\left(t, x_{k}(t), \xi_{k}(t), \lambda_{k}(t)\right) d t \geq \int_{0}^{T} l\left(t, x_{0}(t), \xi_{0}(t), \lambda_{0}(t)\right) d t
$$

for any $T>0$.

Using (4) and (5)

$$
\begin{aligned}
& \liminf _{k \rightarrow \infty} \lim _{T \rightarrow \infty} \int_{0}^{T} l\left(t, x_{k}(t), \xi_{k}(t), \lambda_{k}(t)\right) d t \\
& \quad=\liminf _{S \rightarrow \infty} \liminf _{k \rightarrow \infty} \lim _{T \rightarrow \infty} \int_{0}^{T} l\left(t, x_{k}(t), \xi_{k}(t), \lambda_{k}(t)\right) d t \\
& \quad \geq \liminf _{S \rightarrow \infty} \liminf _{k \rightarrow \infty} \int_{0}^{S} l\left(t, x_{k}(t), \xi_{k}(t), \lambda_{k}(t)\right) d t \\
& \quad \geq \liminf _{S \rightarrow \infty} \int_{0}^{S} l\left(t, x_{0}(t), \xi_{0}(t), \lambda_{0}(t)\right) d t \\
& \quad=\lim _{S \rightarrow \infty} \int_{0}^{S} l\left(t, x_{0}(t), \xi_{0}(t), \lambda_{0}(t)\right) d t .
\end{aligned}
$$

This proves the assertion for $\Psi \equiv 0$ and $M=0$.

Step 2. The general case.

Consider the map

$$
\mathcal{A}(t, x, \xi, \lambda)=l(t, x, \xi, \lambda)-\Phi(t, x, \xi, \lambda)-\Upsilon(t, x, \xi, \lambda)
$$

with

$$
\Phi(t, x, \xi, \lambda)=\Psi(t) \quad \text { and } \quad \Upsilon(t, x, \xi, \lambda)=M \lambda
$$

The function $\mathcal{A}$ satisfies the assumptions of Step 1, much in the same way as in Step 2 of the proof of Theorem 4.2. Hence,

$$
\begin{gathered}
\liminf _{k \rightarrow \infty} \lim _{T \rightarrow \infty} \int_{0}^{T} \mathcal{A}\left(t, x_{k}(t), \xi_{k}(t), \lambda_{k}(t)\right) d t \\
\geq \lim _{T \rightarrow \infty} \int_{0}^{T} \mathcal{A}\left(t, x_{0}(t), \xi_{0}(t), \lambda_{0}(t)\right) d t .
\end{gathered}
$$


As a result,

$$
\begin{aligned}
& \liminf _{k \rightarrow \infty} \lim _{T \rightarrow \infty} \int_{0}^{T} l\left(t, x_{k}(t), \xi_{k}(t), \lambda_{k}(t)\right) d t \\
&=\liminf _{k \rightarrow \infty} \lim _{T \rightarrow \infty} \int_{0}^{T}\left[\left(l\left(t, x_{k}(t), \xi_{k}(t), \lambda_{k}(t)\right)-\Psi(t)-M \lambda_{k}(t)\right)\right. \\
&\left.\quad+\Psi(t)+M \lambda_{k}(t)\right] d t \\
& \geq \liminf _{k \rightarrow \infty} \lim _{T \rightarrow \infty} \int_{0}^{T}\left(l\left(t, x_{k}(t), \xi_{k}(t), \lambda_{k}(t)\right)-\Psi(t)-M \lambda_{k}(t)\right) d t \\
& \quad+\lim _{T \rightarrow \infty} \int_{0}^{T} \Psi(t) d t+\liminf _{k \rightarrow \infty} \lim _{T \rightarrow \infty}^{T} M \lambda_{k}(t) d t \\
& \geq \lim _{T \rightarrow \infty} \int_{0}^{T}\left(l\left(t, x_{0}(t), \xi_{0}(t), \lambda_{0}(t)\right)-\Psi(t)-M \lambda_{0}(t)\right) d t \\
& \quad+\lim _{T \rightarrow \infty} \int_{0}^{T} \Psi(t) d t+\lim _{T \rightarrow \infty} \int_{0}^{T} M \lambda_{0}(t) d t \\
&= \lim _{T \rightarrow \infty} \int_{0}^{T} l\left(t, x_{0}(t), \xi_{0}(t), \lambda_{0}(t)\right) d t .
\end{aligned}
$$

The proof is completed.

\section{Modified Lagrangian}

Let $A:\left[0, \infty\left[\rightrightarrows \mathbb{R}^{n}\right.\right.$ be a multifunction with a closed graph $\mathrm{Gr} A$ and let $R:$ Gr $A \rightrightarrows$ $\mathbb{R}^{1+m}$ be a multifunction.

The multifunction $R$ is said to have property $(K)$ at a point $\left(t, x_{0}\right) \in \operatorname{Gr} A$ with respect to $x$ iff

$$
R\left(t, x_{0}\right)=\bigcap_{\delta>0} \operatorname{cl}\left(\bigcup\left\{R(t, x):\left|x_{0}-x\right|<\delta \wedge x \in A(t)\right\}\right) .
$$

By definition, the multifunction $R$ has property $(K)$ with respect to $x$ iff it has property $(K)$ at each point $\left(t, x_{0}\right) \in \operatorname{Gr} A$ with respect to $x$.

Remark 5.1 If $R$ has property $(K)$ with respect to $x$ then it is obviously closedvalued.

We say that the multifunction $R$ has property $(\Pi)$ iff the fact that $(\eta, \xi) \in R(t, x)$ implies that $(\bar{\eta}, \xi) \in R(t, x)$ for $\bar{\eta} \geq \eta$. 
The modified Lagrangian ${ }^{4}$ is defined to be the function $l:\left[0, \infty\left[\times \mathbb{R}^{n} \times \mathbb{R}^{m+1} \rightarrow\right.\right.$ $\mathbb{R} \cup\{+\infty\}$ given by

$$
l(t, x, \xi, \lambda) \equiv \begin{cases}\inf \{\eta:(\eta, \xi) \in R(t, x), \eta \geq \lambda\}, & \text { if } x \in A(t), \\ +\infty, & \text { if } x \notin A(t)\end{cases}
$$

for any multifunction $R$. By agreement, $\inf \emptyset=+\infty$.

Theorem 5.1 If a multifunction $R$ has the $\mathcal{L}\left(\left[0, \infty[) \times \mathcal{B}\left(\mathbb{R}^{n} \times \mathbb{R}^{m+1}\right)\right.\right.$-measurable graph and enjoys property $(K)$ with respect to $x$ and property $(\Pi)$, then the modified Lagrangian $l$ is a normal integrand on $\left[0, \infty\left[\times\left(\mathbb{R}^{n} \times \mathbb{R}^{m+1}\right)\right.\right.$. Moreover, if $R$ takes convex values, then the function $l(t, x, \cdot, \cdot)$ is convex on $\mathbb{R}^{m+1}$ for any $(t, x) \in \operatorname{Gr} A$.

Proof The $\mathcal{L}\left(\left[0, \infty[) \times \mathcal{B}\left(\mathbb{R}^{n} \times \mathbb{R}^{m+1}\right)\right.\right.$-measurability of $l$ can be proven using the same arguments as in the proof of the measurability of a Lagrangian defined on a bounded interval $[0, T]$, presented in [5]. A proof of the lower semicontinuity of the function $l(t, \cdot, \cdot, \cdot)$ can be found in [14].

\section{Lower Closure Theorems}

We shall prove two lower closure theorems for functions defined on the interval $[0, \infty[$.

\subsection{Case of a (Proper) Lebesgue Integral}

Theorem 6.1 Assume that $A:\left[0, \infty\left[\rightrightarrows \mathbb{R}^{n}\right.\right.$ is a multifunction with a closed graph $\operatorname{Gr} A$ and $R: \operatorname{Gr} A \rightrightarrows \mathbb{R}^{1+m}$ is a convex-valued $\left(\mathcal{L}\left(\left.\left[0, \infty[) \times \mathcal{B}\left(\mathbb{R}^{n}\right)\right)\right|_{\operatorname{Gr} A^{-}}\right.\right.$ measurable multifunction that has property $(K)$ and property $(\Pi)$. Let $\xi_{k}:[0, \infty[\rightarrow$ $\mathbb{R}^{m}, x_{k}:\left[0, \infty\left[\rightarrow \mathbb{R}^{n}, \eta_{k+1}:\left[0, \infty\left[\rightarrow \mathbb{R}\right.\right.\right.\right.$, and $\lambda_{k}:[0, \infty[\rightarrow \mathbb{R}$ be measurable functions for $k \in \mathbb{N} \cup\{0\}$ such that

(i) $x_{k}(t) \in A(t)$ fort $\in[0, \infty[$ a.e. and each $k \in \mathbb{N}$,

(ii) $\left(\eta_{k}(t), \xi_{k}(t)\right) \in R\left(t, x_{k}(t)\right)$ for a.e. $t \in[0, \infty[$ and each $k \in \mathbb{N}$,

(iii) the sequence $\left\{x_{k}\right\}_{k \in \mathbb{N}}$ converges to $x_{0}$ in $L_{\text {loc }}^{1}\left(\left[0, \infty\left[, \mathbb{R}^{n}\right)\right.\right.$, the sequence $\left\{\xi_{k}\right\}_{k \in \mathbb{N}}$ converges weakly to $\xi_{0}$ in $L_{\text {loc }}^{1}\left(\left[0, \infty\left[, \mathbb{R}^{m}\right), \lambda_{k} \in L^{1}([0, \infty[, \mathbb{R})\right.\right.$ for

\footnotetext{
${ }^{4}$ The modified Lagrangian for optimal control problems was introduced by Erik J. Balder in 1982. The classical Lagrangian corresponds to $\lambda=-\infty$ and was used in optimal control problems (see [4]) in connection with the deparameterization procedure. Another similar idea is the Lagrangian auxiliary function which is defined for $(t, x, \xi) \in \operatorname{Gr} A \times \mathbb{R}^{m}$

$$
\mathcal{L}(t, x, \xi):=\operatorname{dist}(\xi, R(t, x))
$$

where dist denotes the Euclidean distance; the details can be found in [5]. It is worth mentioning that there is a close relationship between an auxiliary function and a separation function, which has been considered in [15, Chap. 5].
} 
$k=0,1, \ldots$, the sequence $\left\{\lambda_{k}\right\}_{k \in \mathbb{N}}$ converges weakly to $\lambda_{0}$ in $L_{\text {loc }}^{1}([0, \infty[, \mathbb{R})$, and

$$
\liminf _{k \rightarrow \infty} \int_{0}^{\infty} \lambda_{k}(t) d t \geq \int_{0}^{\infty} \lambda_{0}(t) d t
$$

(iv) $\eta_{k}(t) \geq \lambda_{k}(t)$ for a.e. $t \in[0, \infty[$ and each $k \in \mathbb{N}$,

(v) $\gamma:=\liminf _{k \rightarrow \infty} \int_{0}^{\infty} \eta_{k}(t) d t \in \mathbb{R}$.

Then $x_{0}(t) \in A(t)$ for a.e. $t \in[0, \infty[$ and there exists a summable function $\eta_{0}:[0, \infty[\rightarrow \mathbb{R}$ such that

$$
\begin{aligned}
& \left(\eta_{0}(t), \xi_{0}(t)\right) \in R\left(t, x_{0}(t)\right), \quad \eta_{0}(t) \geq \lambda_{0}(t) \\
& \text { for a.e. } t \in\left[0, \infty\left[, \quad \text { and } \quad \liminf _{k \rightarrow \infty} \int_{0}^{\infty} \eta_{k}(t) d t \geq \int_{0}^{\infty} \eta_{0}(t) d t .\right.\right.
\end{aligned}
$$

Remark 6.1 Since the multifunction $R$ is $\left(\mathcal{L}\left(\left.\left[0, \infty[) \times \mathcal{B}\left(\mathbb{R}^{n}\right)\right)\right|_{\mathrm{Gr} A}\right.\right.$-measurable, therefore, its graph is $\mathcal{L}\left(\left[0, \infty[) \times \mathcal{B}\left(\mathbb{R}^{n}\right) \times \mathcal{B}\left(\mathbb{R} \times \mathbb{R}^{m}\right)\right.\right.$-measurable. This follows from [13, Theorem 1E, p. 164] and [9, Chap. I, Theorem 7.9, and Exercise 1, p. 325].

Proof of Theorem 6.1 The fact that $x_{0}(t) \in A(t)$ for a.e. $t \in[0, \infty$ [ follows immediately from assumptions (i) and (iii) and from the closedness of the set $A(t)$. Let $l:\left[0, \infty\left[\times \mathbb{R}^{n} \times \mathbb{R}^{m+1} \rightarrow \mathbb{R} \cup\{+\infty\}\right.\right.$ be the modified Lagrangian given by (6). By Theorem $5.1, l$ is normal and $l(t, x, \cdot, \cdot)$ is convex on $\mathbb{R}^{m+1}$ for $(t, x) \in \operatorname{Gr} A$. Moreover, it follows from the definition of $l$ that

$$
l(t, x, \xi, \lambda) \geq \lambda
$$

for $(t, x, \xi, \lambda) \in\left[0, \infty\left[\times \mathbb{R}^{n} \times \mathbb{R}^{m+1}\right.\right.$. Since the assumptions of Theorem 4.2 are satisfied, with $\Psi \equiv 0$ and $M=1$, therefore,

$$
\liminf _{k \rightarrow \infty} \int_{0}^{\infty} l\left(t, x_{k}(t), \xi_{k}(t), \lambda_{k}(t)\right) d t \geq \int_{0}^{\infty} l\left(t, x_{0}(t), \xi_{0}(t), \lambda_{0}(t)\right) d t .
$$

It can be deduced from assumptions (ii), (iv), and (6) that

$$
\eta_{k}(t) \geq l\left(t, x_{k}(t), \xi_{k}(t), \lambda_{k}(t)\right)
$$

for a.e. $t \in[0, \infty[$ and for each $k \in \mathbb{N}$. Hence, by (7),

$$
\begin{aligned}
\liminf _{k \rightarrow \infty} \int_{0}^{\infty} \eta_{k}(t) d t & \geq \liminf _{k \rightarrow \infty} \int_{0}^{\infty} l\left(t, x_{k}(t), \xi_{k}(t), \lambda_{k}(t)\right) d t \\
& \geq \int_{0}^{\infty} l\left(t, x_{0}(t), \xi_{0}(t), \lambda_{0}(t)\right) d t \geq \int_{0}^{\infty} \lambda_{0}(t) d t
\end{aligned}
$$

Further, the last inequality and the summability of the function $\lambda_{0}$ on $[0, \infty[$ imply that

$$
\int_{0}^{\infty} l\left(t, x_{0}(t), \xi_{0}(t), \lambda_{0}(t)\right) d t>-\infty
$$


Put

$$
\eta_{0}(t):=l\left(t, x_{0}(t), \xi_{0}(t), \lambda_{0}(t)\right)
$$

for $t \in[0, \infty[$. By assumption $(v)$ and by (8),

$$
\mathbb{R} \ni \gamma=\liminf _{k \rightarrow \infty} \int_{0}^{\infty} \eta_{k}(t) d t \geq \int_{0}^{\infty} \eta_{0}(t) d t .
$$

In view of (9), this implies the summability of the function $\eta_{0}$ on $\left[0, \infty\left[\right.\right.$. Hence, $\eta_{0}$ is finite a.e. on $[0, \infty[$. As a result,

$$
\left\{\tilde{\eta}:\left(\tilde{\eta}, \xi_{0}(t)\right) \in R\left(t, x_{0}(t)\right), \tilde{\eta} \geq \lambda_{0}(t)\right\} \neq \varnothing
$$

for a.e. $t \in\left[0, \infty\left[\right.\right.$. Hence, for a.e. $t \in\left[0, \infty\left[\right.\right.$ there exists a sequence $\left\{\eta_{k}\right\}_{k \in \mathbb{N}}$, depending on $t$, such that $\left(\eta_{k}, \xi_{0}(t)\right) \in R\left(t, x_{0}(t)\right), \eta_{k} \geq \lambda_{0}(t)$, and $\lim _{k \rightarrow \infty} \eta_{k}=\eta_{0}(t)$. By the closedness of the set $R\left(t, x_{0}(t)\right)$,

$$
R\left(t, x_{0}(t)\right) \ni \lim _{k \rightarrow \infty}\left(\eta_{k}, \xi_{0}(t)\right)=\left(\eta_{0}(t), \xi_{0}(t)\right)
$$

for $t \in[0, \infty[$ a.e. Obviously,

$$
\eta_{0}(t) \geq \lambda_{0}(t)
$$

for $t \in[0, \infty[$ a.e. Moreover, as was proven before,

$$
\liminf _{k \rightarrow \infty} \int_{0}^{\infty} \eta_{k}(t) d t \geq \int_{0}^{\infty} \eta_{0}(t) d t .
$$

The proof is completed.

\subsection{Case of an Improper Lebesgue Integral}

Theorem 6.2 Assume that $A:\left[0, \infty\left[\rightrightarrows \mathbb{R}^{n}\right.\right.$ is a multifunction with a closed graph $\operatorname{Gr} A$ and $R: \operatorname{Gr} A \rightrightarrows \mathbb{R}^{1+m}$ is $\left(\mathcal{L}\left(\left.\left[0, \infty[) \times \mathcal{B}\left(\mathbb{R}^{n}\right)\right)\right|_{\mathrm{Gr} A}\right.\right.$-measurable, takes convex values, and has property $(K)$ and property $(\Pi)$. Let $\xi_{k}:\left[0, \infty\left[\rightarrow \mathbb{R}^{m}\right.\right.$, $x_{k}:\left[0, \infty\left[\rightarrow \mathbb{R}^{n}, \eta_{k+1}:\left[0, \infty\left[\rightarrow \mathbb{R}\right.\right.\right.\right.$, and $\lambda_{k}:[0, \infty[\rightarrow \mathbb{R}$ be measurable functions for $k \in \mathbb{N} \cup\{0\}$ such that

(i) $x_{k}(t) \in A(t)$ for a.e. $t \in[0, \infty[$ and each $k \in \mathbb{N}$,

(ii) $\left(\eta_{k}(t), \xi_{k}(t)\right) \in R\left(t, x_{k}(t)\right)$ for a.e. $t \in[0, \infty[$ and each $k \in \mathbb{N}$,

(iii) the sequence $\left\{x_{k}\right\}_{k \in \mathbb{N}}$ converges to $x_{0}$ in $L_{l o c}^{1}\left(\left[0, \infty\left[, \mathbb{R}^{n}\right)\right.\right.$, the sequence $\left\{\xi_{k}\right\}_{k \in \mathbb{N}}$ converges weakly to $\xi_{0}$ in $L_{\mathrm{loc}}^{1}\left(\left[0, \infty\left[, \mathbb{R}^{m}\right), \lambda_{k} \in L_{\mathrm{loc}}^{1}([0, \infty[, \mathbb{R})\right.\right.$, there exists a limit $\lim _{T \rightarrow \infty} \int_{0}^{T} \lambda_{k}(t) d t>-\infty$ for $k=0,1, \ldots$, the sequence $\left\{\lambda_{k}\right\}_{k \in \mathbb{N}}$ converges weakly to $\lambda_{0}$ in $L_{\mathrm{loc}}^{1}([0, \infty[, \mathbb{R})$, and

$$
\liminf _{k \rightarrow \infty} \lim _{T \rightarrow \infty} \int_{0}^{T} \lambda_{k}(t) d t \geq \lim _{T \rightarrow \infty} \int_{0}^{T} \lambda_{0}(t) d t,
$$


(iv) $\eta_{k}(t) \geq \lambda_{k}(t)$ for a.e. $t \in[0, \infty[$ and each $k \in \mathbb{N}$,

(v) the functions $\eta_{k}$ are locally summable and

$$
\gamma:=\liminf _{k \rightarrow \infty} \lim _{T \rightarrow \infty} \int_{0}^{T} \eta_{k}(t) d t \in \mathbb{R} .
$$

Then $x_{0}(t) \in A(t)$ for a.e. $t \in[0, \infty[$, and there exists a locally summable function $\eta_{0}:[0, \infty[\rightarrow \mathbb{R}$ such that

$$
\begin{aligned}
& \lim _{T \rightarrow \infty} \int_{0}^{T} \eta_{0}(t) d t \in \mathbb{R},\left(\eta_{0}(t), \xi_{0}(t)\right) \in R\left(t, x_{0}(t)\right), \\
& \eta_{0}(t) \geq \lambda_{0}(t) \quad \text { for a.e. } t \in[0, \infty), \\
& \text { and } \liminf _{k \rightarrow \infty} \lim _{T \rightarrow \infty} \int_{0}^{T} \eta_{k}(t) d t \geq \lim _{T \rightarrow \infty} \int_{0}^{T} \eta_{0}(t) d t .
\end{aligned}
$$

Remark 6.2 Observe that the existence of the $\operatorname{limit} \lim _{T \rightarrow \infty} \int_{0}^{T} \eta_{k}(t) d t$ for $k \in \mathbb{N}$ follows from assumption (iv), the local summability of $\lambda_{k}$, and the existence of the limit $\lim _{T \rightarrow \infty} \int_{0}^{T} \lambda_{k}(t) d t$ for $k \in \mathbb{N}$.

Proof The proof of Theorem 6.2 is based on Theorem 5.1. It is essentially the same as the proof of Theorem 6.1. The local summability of the function $\eta_{0}(t)=$ $l\left(t, x_{0}(t), \xi_{0}(t), \lambda_{0}(t)\right)$ on $\left[0, \infty\left[\right.\right.$ and the existence of the $\operatorname{limit}_{\lim } \rightarrow \infty \int_{0}^{T} \eta_{0}(t) d t \in$ $\mathbb{R}$ follow from the inequalities

$$
\eta_{0}(t) \geq \lambda_{0}(t) \text { for a.e. } t \in\left[0, \infty\left[\quad \text { and } \quad \lim _{T \rightarrow \infty} \int_{0}^{T} \eta_{0}(t) d t \leq \gamma\right.\right.
$$

and from the fact that the function $\lambda_{0}$ is locally summable, and there exists a limit $\lim _{T \rightarrow \infty} \int_{0}^{T} \lambda_{0}(t) d t>-\infty$.

\section{Existence of a Classical Optimal Pair}

This section contains the main results of the paper.

Let us consider the infinite-horizon optimal control system $(\mathrm{P})$ with the cost functional $\left(J_{\int}\right)$. For this system, we introduce the definition of an admissible pair and of a classical optimal solution.

Assume that

(I1) the multifunctions $A:\left[0, \infty\left[\rightrightarrows \mathbb{R}^{n}\right.\right.$ and $U:$ Gr $A \rightrightarrows \mathbb{R}^{m}$ have closed graphs Gr $A$ and $\operatorname{Gr} U$;

(I2) the set $\bigcup_{x \in Z} U(t, x)$ is bounded for each point $t \in[0, \infty[$ and each bounded set $Z \subset \mathbb{R}^{n}$; 
(I3) $f:\left[0, \infty\left[\times \mathbb{R}^{n} \times \mathbb{R}^{m} \rightarrow \mathbb{R}^{n}\right.\right.$ is a Carathéodory function ${ }^{5}$ with respect to $t \in$ $\left[0, \infty\left[\right.\right.$ and $(x, u) \in \mathbb{R}^{n} \times \mathbb{R}^{m}$ and satisfies the following growth condition: for any $T>0$ there exist a nonnegative summable function $\Psi_{T}:[0, T] \rightarrow \mathbb{R}$ and a constant $A \geq 0$ such that

$$
|f(t, x, u)| \leq \Psi_{T}(t)+A|x|
$$

for each $(t, x, u) \in \mathrm{Gr} U$;

(I4) $F:\left[0, \infty\left[\times \mathbb{R}^{n} \times \mathbb{R}^{m} \rightarrow \mathbb{R}\right.\right.$ is a Carathéodory function with respect to $t \in$ $\left[0, \infty\left[\right.\right.$ and $(x, u) \in \mathbb{R}^{n} \times \mathbb{R}^{m}$

(I5) the multifunction $Q: \operatorname{Gr} A \rightrightarrows \mathbb{R}^{1+n}$ given by

$$
\begin{aligned}
& Q(t, x):=\{(\eta, \xi): \text { there exists a } u \in U(t, x) \\
&\text { such that } \eta \geq F(t, x, u), \xi=f(t, x, u)\}
\end{aligned}
$$

takes convex values.

The following elementary result holds true:

Theorem 7.1 If assumption (I1) is satisfied, then the multifunction $U: \mathrm{Gr} A \rightrightarrows \mathbb{R}^{m}$ is $\left(\mathcal{L}\left(\left.\left[0, \infty[) \times \mathcal{B}\left(\mathbb{R}^{n}\right)\right)\right|_{\text {Gr } A}\right.\right.$-measurable.

Proof Notice that if $\operatorname{Gr} U$ is a closed set, then the set $U^{-1}(C)$ is closed in Gr $A$ for every compact set $C \subset \mathbb{R}^{m}$; see [13, p. 165]. Next, consider an arbitrary compact set $C \subset \mathbb{R}^{m}$. Since the set $U^{-1}(C)$ is closed in $\left[0, \infty\left[\times \mathbb{R}^{n}\right.\right.$, therefore, it is $\mathcal{B}\left(\left[0, \infty\left[\times \mathbb{R}^{n}\right)\right.\right.$-measurable and consequently $\mathcal{L}\left(\left[0, \infty[) \times \mathcal{B}\left(\mathbb{R}^{n}\right)\right.\right.$-measurable. Hence, $U^{-1}(C)$ is an $\left(\mathcal{L}\left(\left.\left[0, \infty[) \times \mathcal{B}\left(\mathbb{R}^{n}\right)\right)\right|_{\mathrm{Gr} A}\right.\right.$-measurable set. By [13, Proposition $1 \mathrm{~A}, \mathrm{p} .160]$, the closedness of $\mathrm{Gr} U$ implies the closedness of the values of the map $U$, which permits us to infer that the map $U$ is $\left(\mathcal{L}\left(\left.\left[0, \infty[) \times \mathcal{B}\left(\mathbb{R}^{n}\right)\right)\right|_{\operatorname{Gr} A^{-}}\right.\right.$ measurable.

The measurability part of Theorem 7.2 has been proven in [5]. That proof is based on the Castaing representation theorem for multifunctions. The proof presented in this paper is based on properties of some special multifunctions. Property $(K)$ of $Q$ may be deduced from the fact that the modified Lagrangian is a normal integrand. We shall prove this property in a direct way.

Theorem 7.2 If assumptions (I1)-(I5) are satisfied, then the multifunction $Q$ : $\operatorname{Gr} A \rightrightarrows \mathbb{R}^{1+n}$ given by $(10)$ is $\left(\mathcal{L}\left(\left.\left[0, \infty[) \times \mathcal{B}\left(\mathbb{R}^{n}\right)\right)\right|_{\mathrm{Gr} A}\right.\right.$-measurable and has property $(K)$ and property $(\Pi)$.

Proof Consider a multifunction $\widetilde{Q}:$ Gr $A \rightrightarrows \mathbb{R}^{1+n+m}$ given by

$$
\widetilde{Q}(t, x)=\{(\eta, \xi, u): u \in U(t, x), \eta \geq F(t, x, u), \xi=f(t, x, u)\} .
$$

\footnotetext{
${ }^{5}$ A function $f:\left[0, \infty\left[\times \mathbb{R}^{n} \times \mathbb{R}^{m} \rightarrow \mathbb{R}^{n}\right.\right.$ is said to be a Carathéodory function iff $f(\cdot, x, u)$ is measurable for any $(x, u) \in \mathbb{R}^{n} \times \mathbb{R}^{m}$ and $f(t, \cdot, \cdot)$ is continuous for any $t \in[0, \infty[$.

${ }^{6} U^{-1}(C):=\{(t, x) \in \mathrm{Gr} A: U(t, x) \cap C \neq \emptyset\}$.
} 
Next, let $\widetilde{Q}_{1}: \operatorname{Gr} A \rightrightarrows \mathbb{R}^{1+n+m}, \widetilde{Q}_{2}: \operatorname{Gr} A \rightrightarrows \mathbb{R}^{1+n+m}$, and $\widetilde{Q}_{3}:$ Gr $A \rightrightarrows \mathbb{R}^{1+n+m}$ be given by

$$
\begin{aligned}
& \widetilde{Q}_{1}(t, x)=\left\{(\eta, \xi, u): f_{1}(t, x, \eta, \xi, u) \in U(t, x)\right\}, \\
& \widetilde{Q}_{2}(t, x)=\left\{(\eta, \xi, u): f_{2}(t, x, \eta, \xi, u) \leq 0\right\}, \\
& \widetilde{Q}_{3}(t, x)=\left\{(\eta, \xi, u): f_{3}(t, x, \eta, \xi, u)=0\right\},
\end{aligned}
$$

where

$$
\begin{aligned}
& f_{1}: \operatorname{Gr} A \times \mathbb{R}^{1+n+m} \rightarrow \mathbb{R}^{m} \text { and } f_{1}(t, x, \eta, \xi, u)=u, \\
& f_{2}: \operatorname{Gr} A \times \mathbb{R}^{1+n+m} \rightarrow \mathbb{R} \text { and } f_{2}(t, x, \eta, \xi, u)=F(t, x, u)-\eta, \\
& f_{3}: \operatorname{Gr} A \times \mathbb{R}^{1+n+m} \rightarrow \mathbb{R}^{n} \text { and } f_{3}(t, x, \eta, \xi, u)=f(t, x, u)-\xi .
\end{aligned}
$$

It follows from the continuity of the function $f_{1}$ that it is a Carathéodory function with respect to $(t, x) \in \operatorname{Gr} A$ and $(\eta, \xi, u) \in \mathbb{R}^{1+n+m}$, i.e., the function $f_{1}(\cdot, \cdot, \eta, \xi, u)$ is measurable with respect to the $\sigma$-algebra $\left(\mathcal{L}\left(\left.\left[0, \infty[) \times \mathcal{B}\left(\mathbb{R}^{n}\right)\right)\right|_{\mathrm{Gr} A}\right.\right.$ for each $(\eta, \xi, u) \in \mathbb{R}^{1+n+m}$, and the function $f_{1}(t, x, \cdot, \cdot, \cdot)$ is continuous on $\mathbb{R}^{1+n+m}$ for each $(t, x) \in \mathrm{Gr} A$. It can be inferred from Theorem 7.1 that the map $U: \operatorname{Gr} A \rightrightarrows \mathbb{R}^{m}$ is measurable. Since the graph $\operatorname{Gr} U$ is closed, the values of $U$ are closed. Hence, by [13, Corollary 1Q], the map $\widetilde{Q}_{1}$ is $\left(\mathcal{L}\left(\left.\left[0, \infty[) \times \mathcal{B}\left(\mathbb{R}^{n}\right)\right)\right|_{\mathrm{Gr} A}\right.\right.$-measurable and takes closed values.

Consider the map

$$
\operatorname{Gr} A \times \mathbb{R}^{1+n+m} \ni(t, x, \eta, \xi, u) \rightarrow F(t, x, u) \in \mathbb{R} .
$$

It can be deduced from assumption (I4) and [13, Proposition 2A and Proposition 2C] that this map is a Carathéodory function with respect to $(t, x) \in \operatorname{Gr} A$ and $(\eta, \xi, u) \in$ $\mathbb{R} \times \mathbb{R}^{n} \times \mathbb{R}^{m}$-and so is the map $f_{2}$, as a consequence. It means that the map $\widetilde{Q}_{2}$ is $\left(\mathcal{L}\left(\left.\left[0, \infty[) \times \mathcal{B}\left(\mathbb{R}^{n}\right)\right)\right|_{\mathrm{Gr} A}\right.\right.$-measurable and closed-valued by [13, Theorem $\left.2 \mathrm{I}\right]$; the fact that $f_{2}$ is a normal integrand follows from [13, Theorem 2C]. Similarly, the map $f_{3}$ is a Carathéodory function with respect to $(t, x) \in \operatorname{Gr} A$ and $(\eta, \xi, u) \in \mathbb{R} \times$ $\mathbb{R}^{n} \times \mathbb{R}^{m}$.

Consider, for $i=1, \ldots, n$, the maps

$$
\widetilde{Q}_{3}^{i}: \operatorname{Gr} A \rightrightarrows \mathbb{R} \times \mathbb{R}^{n+m} \quad \text { and } \quad \widetilde{P}_{3}^{i}: \operatorname{Gr} A \rightrightarrows \mathbb{R} \times \mathbb{R}^{n+m}
$$

given by

$$
\begin{aligned}
& \widetilde{Q}_{3}^{i}(t, x)=\left\{(\eta, \xi, u): f_{3}^{i}(t, x, \eta, \xi, u) \leq 0\right\}, \\
& \widetilde{P}_{3}^{i}(t, x)=\left\{(\eta, \xi, u):-f_{3}^{i}(t, x, \eta, \xi, u) \leq 0\right\},
\end{aligned}
$$

where $f_{3}^{i}$ is the $i$ th coordinate function of $f_{3}$. It follows from [13, Theorem 2I] that the maps $\widetilde{Q}_{3}^{i}$ and $\widetilde{P}_{3}^{i}$ are $\left(\mathcal{L}\left(\left.\left[0, \infty[) \times \mathcal{B}\left(\mathbb{R}^{n}\right)\right)\right|_{\mathrm{Gr} A}\right.\right.$-measurable and closed-valued. 
Thus, the map

$$
\widetilde{Q}_{3}(t, x)=\bigcap_{i=1}^{n}\left(\widetilde{Q}_{3}^{i}(t, x) \cap \widetilde{P}_{3}^{i}(t, x)\right)
$$

is $\left(\mathcal{L}\left(\left.\left[0, \infty[) \times \mathcal{B}\left(\mathbb{R}^{n}\right)\right)\right|_{\mathrm{Gr} A}\right.\right.$-measurable and closed-valued by [13, Corollary $\left.1 \mathrm{M}\right]$. Obviously,

$$
\widetilde{Q}(t, x)=\widetilde{Q}_{1}(t, x) \cap \widetilde{Q}_{2}(t, x) \cap \widetilde{Q}_{3}(t, x)
$$

for $(t, x) \in \operatorname{Gr}(A)$. Consequently, [13, Corollary 1M] implies that the map $\widetilde{Q}$ is $\left(\mathcal{L}\left(\left.\left[0, \infty[) \times \mathcal{B}\left(\mathbb{R}^{n}\right)\right)\right|_{\text {Gr } A}\right.\right.$-measurable and closed-valued.

Now choose any $(t, x) \in \operatorname{Gr} A$ and consider the continuous map $G_{(t, x)}: \mathbb{R} \times$ $\mathbb{R}^{n+m} \rightarrow \mathbb{R} \times \mathbb{R}^{n}$ given by

$$
G_{(t, x)}(\eta, \xi, u)=(\eta, \xi) .
$$

It follows from the continuity of $G_{(t, x)}$ that its graph is closed. As a result, the graph of the multifunction

$$
\widetilde{G}_{(t, x)}: \mathbb{R} \times \mathbb{R}^{n+m} \ni(\eta, \xi, u) \rightrightarrows\{(\eta, \xi)\} \in \mathbb{R} \times \mathbb{R}^{n}
$$

is closed. Further, the constant multifunction

$$
\mathrm{Gr} A \ni(t, x) \rightrightarrows \mathrm{Gr} \widetilde{G}_{(t, x)} \in \mathbb{R} \times \mathbb{R}^{n+m} \times \mathbb{R} \times \mathbb{R}^{n}
$$

is closed-valued and $\left(\mathcal{L}\left(\left.\left[0, \infty[) \times \mathcal{B}\left(\mathbb{R}^{n}\right)\right)\right|_{\mathrm{Gr} A}\right.\right.$-measurable; see [13, Proposition $\left.1 \mathrm{~A}\right]$. It can be inferred directly from the definitions that

$$
Q(t, x)=\widetilde{G}_{(t, x)}(\widetilde{Q}(t, x))
$$

for $(t, x) \in \operatorname{Gr} A$. Since the set $U(t, x)$ is compact (see assumptions (I1) and (I2)), therefore, the set $\widetilde{G}_{(t, x)}(\widetilde{Q}(t, x))$ is closed, i.e.,

$$
Q(t, x)=\operatorname{cl} \widetilde{G}_{(t, x)}(\widetilde{Q}(t, x))
$$

for $(t, x) \in \operatorname{Gr} A$. By [13, Theorem $1 \mathrm{~N}]$, applied to the maps $\widetilde{Q}$ and $\widetilde{G}_{(t, x)}$, the map $Q$ is $\left(\mathcal{L}\left(\left.\left[0, \infty[) \times \mathcal{B}\left(\mathbb{R}^{n}\right)\right)\right|_{\text {Gr } A}\right.\right.$-measurable and closed-valued.

We shall show that the multifunction $Q$ has property $(K)$. Indeed, consider an arbitrary point $\left(t, x_{0}\right) \in \operatorname{Gr} A$. Obviously,

$$
Q\left(t, x_{0}\right) \subseteq \bigcap_{\delta>0} \operatorname{cl}\left(\bigcup\left\{Q(t, x):\left|x-x_{0}\right|<\delta \wedge x \in A(t)\right\}\right) .
$$

To prove the reverse inclusion, consider an arbitrary point $(\eta, \xi) \in \mathbb{R} \times \mathbb{R}^{n}$ such that

$$
(\eta, \xi) \in \bigcap_{\delta>0} \operatorname{cl}\left(\bigcup\left\{Q(t, x):\left|x-x_{0}\right|<\delta \wedge x \in A(t)\right\}\right)
$$


Then

$$
(\eta, \xi) \in \operatorname{cl}\left(\bigcup\left\{Q(t, x):\left|x-x_{0}\right|<\delta \wedge x \in A(t)\right\}\right)
$$

for any $\delta>0$. Hence, for any $k \in \mathbb{N}$ there exists a point

$$
\left(\eta_{k}, \xi_{k}\right) \in \bigcup\left\{Q(t, x):\left|x-x_{0}\right|<\frac{1}{k} \wedge x \in A(t)\right\}
$$

such that $\left|(\eta, \xi)-\left(\eta_{k}, \xi_{k}\right)\right|<\frac{1}{k}$. As a result,

$$
\lim _{k \rightarrow \infty}\left(\eta_{k}, \xi_{k},\right)=(\eta, \xi)
$$

and, for any $k \in \mathbb{N}$, there exists $x_{k}$ such that

$$
\left|x_{k}-x_{0}\right|<\frac{1}{k} \wedge x_{k} \in A(t) \wedge\left(\eta_{k}, \xi_{k}\right) \in Q\left(t, x_{k}\right) .
$$

Hence, the sequence $\left\{x_{k}\right\}_{k \in \mathbb{N}}$ converges to $x_{0}$. It follows from the closedness of $\operatorname{Gr} A$ that $x_{0} \in A(t)$. Next, by the definition of $Q$ there exists a sequence $\left\{u_{k}\right\}_{k \in \mathbb{N}}$ such that

$$
u_{k} \in U\left(t, x_{k}\right), \quad \eta_{k} \geq F\left(t, x_{k}, u_{k}\right), \text { and } \xi_{k}=f\left(t, x_{k}, u_{k}\right)
$$

for any $k \in \mathbb{N}$. It can be inferred from the boundedness of the sequence $\left\{x_{k}\right\}_{k \in \mathbb{N}}$ and from assumption (I2) that there exists a subsequence of the sequence $\left\{u_{k}\right\}_{k \in \mathbb{N}}$, still denoted by $\left\{u_{k}\right\}_{k \in \mathbb{N}}$, that converges to some $u_{0}$. It follows from the closedness of the graph Gr $U$ and the convergence of the sequence $\left\{x_{k}\right\}_{k \in \mathbb{N}}$ that $u_{0} \in U\left(t, x_{0}\right)$. The continuity of the function $F(t, \cdot, \cdot)$ implies

$$
\eta=\lim _{k \rightarrow \infty} \eta_{k} \geq \lim _{k \rightarrow \infty} F\left(t, x_{k}, u_{k}\right)=F\left(t, x_{0}, u_{0}\right) .
$$

The continuity of the function $f(t, \cdot, \cdot)$ leads to the conclusion that

$$
\xi=\lim _{k \rightarrow \infty} \xi_{k}=\lim _{k \rightarrow \infty} f\left(t, x_{k}, u_{k}\right)=f\left(t, x_{0}, u_{0}\right) .
$$

Thus, $(\eta, \xi) \in Q\left(t, x_{0}\right)$, which means that $Q$ has property $(K)$.

The fact that $Q$ has property $(\Pi)$ follows immediately from the definition of $Q$.

Using Gronwall's lemma, one can prove the following.

Theorem 7.3 If a function $f:\left[0, \infty\left[\times \mathbb{R}^{n} \times \mathbb{R}^{m} \rightarrow \mathbb{R}^{n}\right.\right.$ satisfies assumption (I3), then the set of all admissible trajectories is a relatively sequentially weakly compact subset of $A C_{\mathrm{loc}}\left(\left[0, \infty\left[, \mathbb{R}^{n}\right)\right.\right.$ (this set may be empty).

Remark 7.1 A similar theorem has been proven in [3] under a stronger assumption on $f$, the so-called growth condition $(\gamma)$. The set of all admissible trajectories is nonempty under that assumption; see [6]. By applying an analogous method, we can obtain the compactness of the set of admissible trajectories under the weaker assumption (I3). However, (I3) does not ensure that the set of all admissible trajectories is nonempty. 


\subsection{Existence of a Classical Optimal Solution}

In this section, we can state and prove a theorem on the existence of a classical optimal solution to the problem described by $(\mathrm{P})$ and $\left(J_{\int}\right)$.

Definition 7.4 A pair of functions $(x, u):\left[0, \infty\left[\rightarrow \mathbb{R}^{n} \times \mathbb{R}^{m}\right.\right.$ is called admissible for the optimal control problem given by $(\mathrm{P})$ and $\left(J_{f}\right)$ if $x \in A C_{\mathrm{loc}}\left(\left[0, \infty\left[, \mathbb{R}^{n}\right), u\right.\right.$ is measurable, the pair $(x, u)$ satisfies system $(\mathrm{P})$, and the function $F(\cdot, x(\cdot), u(\cdot))$ is integrable on $[0, \infty[$ (not necessarily summable).

The set of all admissible pairs $(x, u)$ introduced in Definition 7.4 is denoted by $\Omega_{\int}$. A function $x \in A C_{\text {loc }}\left(\left[0, \infty\left[, \mathbb{R}^{n}\right)\right.\right.$ is called an admissible trajectory if there exists a measurable function $u$ such that $(x, u) \in \Omega_{\int}$.

Definition 7.5 A pair $\left(x^{*}, u^{*}\right) \in \Omega_{\int}$ is called a classical optimal solution to the problem given by $(\mathrm{P})$ and $\left(J_{\int}\right)$ if $J_{f}\left(x^{*}, u^{*}\right) \in \mathbb{R}$ and

$$
J_{\int}\left(x^{*}, u^{*}\right) \leq J_{\int}(x, u)
$$

for each pair $(x, u) \in \Omega_{\int}$.

Assume that:

(If 6$)$ there exists a constant $\alpha \in \mathbb{R}$ such that

$$
\Omega_{\int}^{\alpha}:=\left\{(x, u) \in \Omega_{\int}: J_{\int}(x, u) \leq \alpha\right\} \neq \emptyset,
$$

$\left(I_{f} 7\right)$ there exists a summable function $\lambda:[0, \infty[\rightarrow \mathbb{R}$ such that for each pair $(x, u) \in \Omega_{f}^{\alpha}$

$$
F(t, x(t), u(t)) \geq \lambda(t) \quad \text { for a.e. } t \in[0, \infty[\text {. }
$$

Theorem 7.6 If assumptions $(I 1)-(I 5),\left(I_{f} 6\right)$, and $\left(I_{f} 7\right)$ are satisfied, then the problem given by $(\mathrm{P})$ and $\left(J_{f}\right)$ has a classical optimal solution.

Proof Assumption $\left(I_{f} 6\right)$ implies that

$$
l:=\inf _{(x, u) \in \Omega_{f}} J_{f}(x, u)=\inf _{(x, u) \in \Omega_{f}^{\alpha}} J_{f}(x, u) \leq \alpha .
$$

Consequently, by $\left(I_{f} 7\right)$

$$
\mathbb{R} \ni \int_{0}^{\infty} \lambda(s) d s \leq l \leq \alpha .
$$

Let $\left\{\left(x_{k}, u_{k}\right)\right\}_{k \in \mathbb{N}} \subset \Omega_{\int}^{\alpha}$ be such a sequence that

$$
\lim _{k \rightarrow \infty} J_{\int}\left(x_{k}, u_{k}\right)=l
$$


By Theorem 7.3, one can choose a subsequence of the sequence $\left\{x_{k}\right\}_{k \in \mathbb{N}}$, that converges weakly in $A C_{\text {loc }}\left(\left[0, \infty\left[, \mathbb{R}^{n}\right)\right.\right.$ to some $x^{*} \in A C_{\text {loc }}\left(\left[0, \infty\left[, \mathbb{R}^{n}\right)\right.\right.$. Without loss of generality, this subsequence shall be denoted by $\left\{x_{k}\right\}_{k \in \mathbb{N}}$. Define

$$
\begin{aligned}
& \eta_{k}(t)=F\left(t, x_{k}(t), u_{k}(t)\right) \text { for a.e. } t \in[0, \infty[, k \in \mathbb{N}, \\
& x_{0}(t)=x^{*}(t) \text { for } t \in[0, \infty[, \\
& \xi_{k}(t)=\dot{x}_{k}(t) \text { for a.e. } t \in[0, \infty[, k \in \mathbb{N} \cup\{0\}, \\
& \lambda_{k}(t)=\lambda(t) \text { for a.e. } t \in[0, \infty[, k \in \mathbb{N} \cup\{0\} .
\end{aligned}
$$

Since $\left\{\left(x_{k}, u_{k}\right)\right\}_{k \in \mathbb{N}} \subset \Omega_{\int}^{\alpha}$, the functions $\eta_{k}$ are summable on $[0, \infty[$ for $k \in \mathbb{N}$ and $\liminf _{k \rightarrow \infty} \int_{0}^{\infty} \eta_{k}(t) d t \in \mathbb{R}$. By Theorem 3.3(i), the sequence $\left\{x_{k}(t)\right\}_{k \in \mathbb{N}}$ converges to $x^{*}(t)$ for any $t \in[0, \infty$ [. Using the closedness of the graph $\operatorname{Gr} A$ and the fact that $x_{k}(t) \in A(t)$ for $t \in[0, \infty[, k \in \mathbb{N}$, it can be deduced that

$$
x^{*}(t) \in A(t) \quad \text { for } t \in[0, \infty[.
$$

In view of Theorem 3.2(i), the sequence $\left\{\left.\dot{x}_{k}\right|_{D_{T}}\right\}_{k \in \mathbb{N}}$ converges weakly to $\left.\dot{x}\right|_{D_{T}}$ in $L^{1}\left([0, T], \mathbb{R}^{n}\right)$ for any $T>0$. The multifunction $Q$ satisfies the assumptions of Theorem 6.1 that concern $R$ (this follows from Theorem 7.2 and assumption (I5)) and the sequence $\left\{\left(\eta_{k}, \xi_{k}\right)\right\}_{k \in \mathbb{N}}$ satisfies condition (ii) of Theorem 6.1. The constant sequence $\left\{\lambda_{k}\right\}_{k \in \mathbb{N}}$ converges weakly to $\lambda_{0}=\lambda$ in $L_{\text {loc }}^{1}([0, \infty[, \mathbb{R})$ in an obvious way and

$$
\liminf _{k \rightarrow \infty} \int_{0}^{\infty} \lambda_{k}(t) d t \geq \int_{0}^{\infty} \lambda_{0}(t) d t .
$$

By $\left(I_{f} 7\right), \eta_{k}(t) \geq \lambda_{k}(t)$ for a.e. $t \in[0, \infty[$ and $k \in \mathbb{N}$. Applying Theorem 3.3(ii) leads to the conclusion that the sequence $\left\{x_{k}\right\}_{k \in \mathbb{N}}$ converges to $x_{0}$ in $L_{\text {loc }}^{1}([0, \infty[, \mathbb{R})$. Thus, the assumptions of Theorem 6.1 are satisfied. Consequently, there exists a summable function $\eta_{0}:[0, \infty[\rightarrow \mathbb{R}$ such that

$$
\begin{aligned}
& \liminf _{k \rightarrow \infty} \int_{0}^{\infty} \eta_{k}(t) d t \geq \int_{0}^{\infty} \eta_{0}(t) d t \\
& \eta_{0}(t) \geq \lambda(t) \text { and }\left(\eta_{0}(t), \xi_{0}(t)\right) \in Q\left(t, x^{*}(t)\right) \text { for a.e. } t \in[0, \infty[.
\end{aligned}
$$

Now consider the multifunction $\Gamma:\left[0, \infty\left[\rightrightarrows \mathbb{R}^{m}\right.\right.$ given by

$$
\Gamma(t)=\left\{u: u \in U\left(t, x^{*}(t)\right), \eta_{0}(t) \geq F\left(t, x^{*}(t), u\right), \dot{x}(t)=f\left(t, x^{*}(t), u\right)\right\} .
$$

Note that the closed-valued multifunction $\left[0, \infty\left[\ni t \rightrightarrows U\left(t, x^{*}(t)\right) \in \mathbb{R}^{m}\right.\right.$ is measurable; the closedness of the values follows from the closedness of the graph $\operatorname{Gr} U$. Indeed, consider the closed-valued multifunction $\bar{\Gamma}:\left[0, \infty\left[\rightrightarrows \mathbb{R}^{1+n}\right.\right.$ given by

$$
\bar{\Gamma}(t)=\left\{\left(t, x^{*}(t)\right)\right\}, \quad t \in[0, \infty[.
$$


The measurability of this multifunction follows from the continuity of the function $\left[0, \infty\left[\ni t \rightarrow\left(t, x^{*}(t)\right) \in \mathbb{R} \times \mathbb{R}^{n}\right.\right.$. Next, for any fixed $t \in[0, \infty[$, consider the multifunction $A_{t}: \mathbb{R} \times \mathbb{R}^{n} \rightrightarrows \mathbb{R}^{m}$ given by

$$
A_{t}(s, x)= \begin{cases}U(s, x), & (s, x) \in \mathrm{Gr} A, \\ \emptyset, & (s, x) \notin \mathrm{Gr} A .\end{cases}
$$

It is easy to see that

$$
\operatorname{Gr} A_{t}=\operatorname{Gr} U \text {. }
$$

Hence, Gr $A_{t}$ is closed. Moreover, the multifunction

$$
\left[0, \infty\left[\ni t \rightrightarrows \mathrm{Gr} A_{t} \in \mathbb{R} \times \mathbb{R}^{n} \times \mathbb{R}^{m}\right.\right.
$$

is measurable as a constant closed-valued map; see [13, Proposition 1A]. By [13, Theorem $1 \mathrm{~N}]$, the multifunction

$$
\left[0, \infty\left[\ni t \rightrightarrows \mathrm{cl} A_{t}(\bar{\Gamma}(t))=U\left(t, x^{*}(t)\right) \in 2^{\mathbb{R}^{m}}\right.\right.
$$

is measurable. In view of [13, Theorem $2 \mathrm{~N}$ and Proposition $2 \mathrm{C}]$, the map $G$ : $\left[0, \infty\left[\times \mathbb{R}^{m} \rightarrow \mathbb{R}\right.\right.$ given by

$$
G(t, u)=F\left(t, x^{*}(t), u\right)
$$

is a normal integrand and the map $H:\left[0, \infty\left[\times \mathbb{R}^{m} \rightarrow \mathbb{R}^{n}\right.\right.$ given by

$$
H:(t, u) \rightarrow f\left(t, x^{*}(t), u\right)
$$

is a Carathéodory function. Since $\left(\eta_{0}(t), \xi_{0}(t)\right) \in Q\left(t, x^{*}(t)\right)$ for a.e. $t \in[0, \infty[$, therefore, the map $\Gamma$ has nonempty values a.e. on $[0, \infty[$. Using the implicit function theorem for multifunctions ([13, Theorem $2 \mathrm{~J}]$ ), notice that the map $\Gamma$ is measurable, closed-valued, and there exists a measurable function $u^{*}:\left[0, \infty\left[\rightarrow \mathbb{R}^{m}\right.\right.$ such that $u^{*}(t) \in \Gamma(t)$ for a.e. $t \in[0, \infty[$. It follows from the description of $\Gamma$ that

$$
\left(\dot{x}^{*}\right)(t)=f\left(t, x^{*}(t), u^{*}(t)\right), \quad u^{*}(t) \in U\left(t, x^{*}(t)\right) \quad \text { for a.e. } t \in[0, \infty[\text {. }
$$

The pointwise convergence of admissible trajectories $x_{k}(t)$ to $x^{*}(t)$ on $[0, \infty[$ and the initial conditions $x_{k}(0)=0$ for $k \in \mathbb{N}$ imply that $x^{*}(0)=0$. As a result, the pair $\left(x^{*}, u^{*}\right)$ satisfies $(\mathrm{P})$. Moreover, it follows from the definition of $\Gamma$ that

$$
\eta_{0}(t) \geq F\left(t, x^{*}(t), u^{*}(t)\right) \text { for a.e. } t \in[0, \infty[\text {. }
$$

Thus, the summability of the function $\eta_{0}$ implies the integrability of the function

$$
\left[0, \infty\left[\ni t \rightarrow F\left(t, x^{*}(t), u^{*}(t)\right) \in \mathbb{R}\right. \text {. }\right.
$$


Finally, $\left(x^{*}, u^{*}\right) \in \Omega_{\int}$. Apply this fact and (11), (13), (14), and (15) to obtain

$$
\begin{aligned}
l & =\lim _{k \rightarrow \infty} \int_{0}^{\infty} F\left(t, x_{k}(t), u_{k}(t)\right) d t=\liminf _{k \rightarrow \infty} \int_{0}^{\infty} F\left(t, x_{k}(t), u_{k}(t)\right) d t \\
& \geq \int_{0}^{\infty} \eta_{0}(t) d t \geq \int_{0}^{\infty} F\left(t, x^{*}(t), u^{*}(t)\right) d t \geq l .
\end{aligned}
$$

Hence, by (12),

$$
\mathbb{R} \ni l=\int_{0}^{\infty} F\left(t, x^{*}(t), u^{*}(t)\right) d t,
$$

and the proof is completed.

Example 7.1 Consider the control system given by

$$
\begin{cases}\dot{x}(t)=a x(t)+b u(t) & \text { for a.e. } t \in[0, \infty[, \\ x(0)=0, & \text { for } t \in[0, \infty[, \\ x(t) \in A(t) & \text { for a.e. } t \in[0, \infty[, \\ u(t) \in U(t, x(t)) & \end{cases}
$$

where $a \in \mathbb{R} \backslash\{0\}, b \in \mathbb{R} \backslash\{0\}, x \in \mathbb{R}, u \in \mathbb{R}, A(t)=\mathbb{R}, U(t, x)=\left[-\frac{1}{t^{2}+1}, \frac{1}{t^{2}+1}\right]$ for $t \in[0, \infty[, x \in \mathbb{R}$. This is a special case of system $(\mathrm{P})$.

It is easy to see that there exists a unique solution $x \in A C_{\text {loc }}([0, \infty[, \mathbb{R})$ of system $\left(\mathrm{P}^{1}\right)$, that corresponds to any fixed measurable control $u:[0, \infty[\rightarrow \mathbb{R}$ such that $u(t) \in\left[-\frac{1}{t^{2}+1}, \frac{1}{t^{2}+1}\right]$ for a.e. $t \in[0, \infty[$.

Assume that the cost functional is given by

$$
J_{f}(x, u)=\int_{0}^{\infty}\left(\frac{\sin ^{2}(x(t)) u^{2}(t)}{1+t^{2}}+u(t)\right) d t .
$$

It is easy to see that the integral $\int_{0}^{\infty}\left(\frac{\sin ^{2}(x(t)) u^{2}(t)}{1+t^{2}}+u(t)\right) d t$ exists and is finite for an arbitrary pair $(x, u)$ such that $x \in A C_{\text {loc }}([0, \infty[, \mathbb{R})$ and $u:[0, \infty[\rightarrow \mathbb{R}$ is a measurable function that satisfies system $\left(\mathrm{P}^{1}\right)$. Hence, $\Omega_{f} \neq \emptyset$.

Moreover, the optimal control problem given by $\left(\mathrm{P}^{1}\right)$ and $\left(J_{f}^{1}\right)$ satisfies the assumptions of Theorem 7.6 with $F(t, x, u)=\frac{\sin ^{2}(x) u^{2}}{1+t^{2}}+u$. Indeed, assumptions (I1)-(I4) are fulfilled in an obvious way. From the convexity of the set $U(t, x)$ and from the convexity of the function,

$$
U(t, x) \ni u \rightarrow \frac{\sin ^{2}(x) u^{2}}{1+t^{2}}+u \in \mathbb{R},
$$

it follows that the set $Q(t, x)$ is convex, i.e., assumption (I5) is satisfied.

Moreover, for $\widetilde{u}(t)=0$

$$
J(\tilde{x}, \tilde{u})=J(0,0)=0
$$


$\tilde{x}=0$ is the unique solution of system $\left(\mathrm{P}^{1}\right)$, corresponding to $\tilde{u}=0$. Hence, $\Omega_{f}^{0} \neq \emptyset$, i.e. assumption $\left(I_{f} 6\right)$ is satisfied with $\alpha=0$. Finally,

$$
\frac{\sin ^{2}(x(t)) u^{2}(t)}{1+t^{2}}+u(t) \geq u(t) \geq-\frac{1}{1+t^{2}} \quad \text { for a.e. } t \in[0, \infty[
$$

for any $(x, u) \in \Omega_{f}^{0}$. Thus, assumption $\left(I_{f} 7\right)$ is satisfied with $\lambda(t)=-\frac{1}{1+t^{2}}$. The function $\left[0, \infty\left[\ni t \rightarrow-\frac{1}{1+t^{2}} \in \mathbb{R}\right.\right.$ is obviously summable. Consequently, the control system given by $\left(\mathrm{P}^{1}\right)$ and $\left(J_{f}^{1}\right)$ has a classical optimal solution $\left(x^{*}, u^{*}\right)$.

\subsection{Existence of an Almost Strongly Optimal Solution}

First, we define an admissible pair and an almost strongly optimal pair for the problem $(\mathrm{P})$, with the functional given by $\left(J_{\lim }\right)$.

Definition 7.7 A pair of functions $(x, u):\left[0, \infty\left[\rightarrow \mathbb{R}^{n} \times \mathbb{R}^{m}\right.\right.$ is called admissible for the optimal control system (P) with the functional $J_{\lim }$ iff $x \in A C_{\operatorname{loc}}\left(\left[0, \infty\left[, \mathbb{R}^{n}\right), u\right.\right.$ is measurable function, the pair $(x, u)$ satisfies system $(\mathrm{P})$, a function $F(t, x(t), u(t))$ is locally integrable on $[0, \infty[$, and there exists a limit

$$
\lim _{T \rightarrow \infty} \int_{0}^{T} F(t, x(t), u(t)) d t
$$

(not necessarily finite).

The set of all admissible pairs $(x, u)$, in the sense of Definition 7.7, will be denoted by $\Omega_{\lim }$. A function $x \in A C_{\mathrm{loc}}\left(\left[0, \infty\left[, \mathbb{R}^{n}\right)\right.\right.$ is called an admissible trajectory iff there exists a measurable function $u$ such that $(x, u) \in \Omega_{\text {lim }}$.

Definition 7.8 A pair $\left(x^{*}, u^{*}\right) \in \Omega_{\lim }$ is called almost strongly optimal iff $J_{\lim }\left(x^{*}, u^{*}\right) \in \mathbb{R}$ and

$$
J_{\lim }\left(x^{*}, u^{*}\right) \leq J_{\lim }(x, u)
$$

for any pair $(x, u) \in \Omega_{\lim }$.

We require that the following conditions hold true:

( $\left.I_{\lim } 6\right)$ there exists a constant $\alpha \in \mathbb{R}$ such that

$$
\Omega_{\lim }^{\alpha}:=\left\{(x, u) \in \Omega_{\lim }: J_{\lim }(x, u) \leq \alpha\right\} \neq \emptyset,
$$

$\left(I_{\lim } 7\right)$ there exists a locally summable function $\lambda:[0, \infty[\rightarrow \mathbb{R}$ such that $\lim _{T \rightarrow \infty} \int_{0}^{T} \lambda(t) d t>-\infty$ and, for any pair $(x, u) \in \Omega_{\lim }^{\alpha}$,

$$
F(t, x(t), u(t)) \geq \lambda(t) \quad \text { for a.e. } t \in[0, \infty[\text {. }
$$


We have the following theorem on the existence of an almost strongly optimal solution.

Theorem 7.9 If assumptions (I1)-(I5), ( $\left.I_{\mathrm{lim}} 6\right)$, and $\left(I_{\mathrm{lim}} 7\right)$ are satisfied, then the problem given by $(\mathrm{P})$ and $\left(J_{\mathrm{lim}}\right)$ has an almost strongly optimal solution .

Proof The fact that $\Omega_{\lim }^{\alpha} \neq \emptyset$ for some $\alpha \in \mathbb{R}$ (see $I_{\text {lim }} 6$ ) implies that

$$
l:=\inf _{(x, u) \in \Omega_{\lim }} J_{\lim }(x, u)=\inf _{(x, u) \in \Omega_{\lim }^{\alpha}} J_{\lim }(x, u) \leq \alpha .
$$

By assumption $\left(I_{\lim } 7\right)$,

$$
-\infty<\lim _{T \rightarrow \infty} \int_{0}^{T} \lambda(t) d t \leq l \leq \alpha .
$$

Let $\left\{\left(x_{k}, u_{k}\right)\right\}_{k \in \mathbb{N}} \subset \Omega_{\lim }^{\alpha}$ be a sequence such that

$$
\lim _{k \rightarrow \infty} J_{\lim }\left(x_{k}, u_{k}\right)=l .
$$

By Theorem 7.3, one can choose a subsequence of the sequence $\left\{x_{k}\right\}_{k \in \mathbb{N}}$, weakly convergent in $A C_{\text {loc }}\left(\left[0, \infty\left[, \mathbb{R}^{n}\right)\right.\right.$ to some $x^{*} \in A C_{\text {loc }}\left(\left[0, \infty\left[, \mathbb{R}^{n}\right)\right.\right.$. Without loss of generality, we shall denote this subsequence by $\left\{x_{k}\right\}_{k \in \mathbb{N}}$. Define the functions $\eta_{k}, x_{0}$, $\zeta_{k}$, and $\lambda_{k}$ as in the proof of Theorem 7.6. Since $\left\{\left(x_{k}, u_{k}\right)\right\}_{k \in \mathbb{N}} \subset \Omega_{\text {lim }}^{\alpha}$, therefore the functions $\eta_{k}$ are locally summable on $[0, \infty[$ for $k \in \mathbb{N}$. It can be checked in the same way as in the proof of Theorem 7.6 that the sequence $\left\{x_{k}(t)\right\}_{k \in \mathbb{N}}$ converges to $x^{*}(t)$ for any $t \in[0, \infty[$,

$$
x^{*}(t) \in A(t) \quad \text { for } t \in[0, \infty[
$$

and the assumptions of Theorem 6.2 are satisfied with $R=Q$ (see (I5)); the fact that

$$
\gamma:=\liminf _{k \rightarrow \infty} \lim _{T \rightarrow \infty} \int_{0}^{T} \eta_{k}(t) d t \in \mathbb{R}
$$

follows from (17) and (18). As a result, there exists a locally summable function $\eta_{0}:[0, \infty[\rightarrow \mathbb{R}$ such that

$$
\begin{aligned}
& \liminf _{k \rightarrow \infty} \lim _{T \rightarrow \infty} \int_{0}^{T} \eta_{k}(t) d t \geq \lim _{T \rightarrow \infty} \int_{0}^{T} \eta_{0}(t) d t \in \mathbb{R}, \\
& \eta_{0}(t) \geq \lambda(t) \text { and }\left(\eta_{0}(t), \xi_{0}(t)\right) \in Q\left(t, x^{*}(t)\right) \text { for a.e. } t \in[0, \infty[.
\end{aligned}
$$

Consider now the multifunction $\Gamma:\left[0, \infty\left[\rightrightarrows \mathbb{R}^{m}\right.\right.$ given by

$$
\Gamma(t)=\left\{u: u \in U\left(t, x^{*}(t)\right), \eta_{0}(t) \geq F\left(t, x^{*}(t), u\right), \dot{x}(t)=f\left(t, x^{*}(t), u\right)\right\} .
$$

It follows from the implicit function theorem for multifunctions [13, Theorem 2J], that there exists a measurable function $u^{*}:\left[0, \infty\left[\rightarrow \mathbb{R}^{m}\right.\right.$ such that $u^{*}(t) \in \Gamma(t)$ for 
a.e. $t \in[0, \infty[$; see the proof of Theorem 7.6 for details. By the description of $\Gamma$,

$$
\begin{aligned}
\left(\dot{x}^{*}\right)(t) & =f\left(t, x^{*}(t), u^{*}(t)\right) \quad \text { for a.e. } t \in[0, \infty[ \\
u^{*} & \in U\left(t, x^{*}(t)\right) \quad \text { for a.e. } t \in[0, \infty[ \\
\eta_{0}(t) & \geq F\left(t, x^{*}(t), u^{*}(t)\right) \quad \text { for a.e. } t \in[0, \infty[.
\end{aligned}
$$

The pointwise convergence of the trajectories $x_{k}(t)$ to $x^{*}(t)$ on $[0, \infty[$ and the conditions $x_{k}(0)=0, k \in \mathbb{N}$, lead to the conclusion that $x^{*}(0)=0$. Thus, the pair $\left(x^{*}, u^{*}\right)$ satisfies system (P); see (19). Since $\eta_{0}$ is locally summable on [0, $\infty[$, (21) implies that the function $F\left(t, x^{*}(t), u^{*}(t)\right)$ is locally integrable on [0, [. Using the fact that $\lim _{T \rightarrow \infty} \int_{0}^{T} \eta_{0}(t) d t \in \mathbb{R}$ and that there exists a limit

$$
\lim _{T \rightarrow \infty} \int_{0}^{T}\left(F\left(t, x^{*}(t), u^{*}(t)\right)-\eta_{0}(t)\right) d t
$$

as a limit of a nonincreasing function (see (21)), we can claim that there exists a limit

$$
\lim _{T \rightarrow \infty} \int_{0}^{T} F\left(t, x^{*}(t), u^{*}(t)\right) d t
$$

finite or equal to $-\infty$. Thus, $\left(x^{*}, u^{*}\right) \in \Omega_{\lim }$. From (16), (18), (20), and (21)

$$
\begin{aligned}
l=\lim _{k \rightarrow \infty} \lim _{T \rightarrow \infty} \int_{0}^{T} F\left(t, x_{k}(t), u_{k}(t)\right) d t & \geq \lim _{T \rightarrow \infty} \int_{0}^{T} \eta_{0}(t) d t \\
& \geq \lim _{T \rightarrow \infty} \int_{0}^{T} F\left(t, x^{*}(t), u^{*}(t)\right) d t \geq l .
\end{aligned}
$$

This means that the pair $\left(x^{*}, u^{*}\right)$ is almost strongly optimal, since by (17)

$$
\mathbb{R} \ni l=\lim _{T \rightarrow \infty} \int_{0}^{T} F\left(t, x^{*}(t), u^{*}(t)\right) d t
$$

The proof is completed.

Example 7.2 Consider a problem

$$
\begin{cases}x^{\prime}(t)=a x(t)+b u(t) & \text { for a.e. } t \in[0, \infty[, \\ x(0)=0, & \text { for } t \in[0, \infty[, \\ x(t) \in A(t) & \text { for a.e. } t \in[0, \infty[, x \in \mathbb{R}, \\ u(t) \in U(t, x(t)) & \end{cases}
$$


where $a \in \mathbb{R} \backslash\{0\}, b \in \mathbb{R} \backslash\{0\}, x \in \mathbb{R}, u \in \mathbb{R}, A(t)=\mathbb{R}$, and

$$
U(t, x)= \begin{cases}{\left[\frac{1}{k+1}, \frac{2}{k+1}\right]} & \text { for } t \in] 2 k, 2 k+1[, k \in \mathbb{N} \cup\{0\}, \\ {\left[-\frac{1}{k+1}, 0\right]} & \text { for } t \in] 2 k+1,2 k+2[, k \in \mathbb{N} \cup\{0\}, \\ {[1,2]} & \text { for } t=0, \\ {\left[-\frac{1}{k+1}, \frac{2}{k+1}\right]} & \text { for } t=2 k+1, k \in \mathbb{N} \cup\{0\}, \\ {\left[-\frac{1}{k}, \frac{2}{k+1}\right]} & \text { for } t=2 k, k \in \mathbb{N}\end{cases}
$$

for $t \in\left[0, \infty\left[\right.\right.$ and $x \in \mathbb{R}$. System $\left(\mathrm{P}^{2}\right)$ is a special case of system $(\mathrm{P})$.

Consider the cost functional given by

$$
J_{\lim }(x, u)=\lim _{T \rightarrow \infty} \int_{0}^{T}\left(\frac{\sin ^{2}(x(t)) u^{2}(t)}{1+t^{2}}+u(t)\right) d t .
$$

System $\left(\mathrm{P}^{2}\right)$, with the functional $\left(J_{\text {lim }}^{2}\right)$, satisfies assumptions $(I 1)-(I 5)$. Consider the control $\tilde{u}:[0, \infty[\rightarrow \mathbb{R}$ given by

$$
\tilde{u}(t)= \begin{cases}\frac{1}{k+1} & \text { for } t \in] 2 k, 2 k+1[, k \in \mathbb{N} \cup\{0\}, \\ -\frac{1}{k+1} & \text { for } t \in] 2 k+1,2 k+2[, k \in \mathbb{N} \cup\{0\} .\end{cases}
$$

The control $\tilde{u}$ and the corresponding trajectory $\tilde{x} \in A C_{\text {loc }}([0, \infty[, \mathbb{R})$ satisfy system $\left(\mathrm{P}^{2}\right)$, the function $F(t, \widetilde{x}(t), \widetilde{u}(t))$ is locally summable on $[0, \infty[$, and there exists

$$
\lim _{T \rightarrow \infty} \int_{0}^{T} F(t, \tilde{x}(t), \tilde{u}(t)) d t .
$$

This means that $(\tilde{x}, \tilde{u}) \in \Omega_{\lim }$.

Moreover,

$$
\begin{aligned}
J_{\lim }(\tilde{x}, \tilde{u}) & =\lim _{T \rightarrow \infty} \int_{0}^{T}\left(\frac{\sin ^{2}(\tilde{x}(t)) \tilde{u}^{2}(t)}{1+t^{2}}+\tilde{u}(t)\right) d t \\
& \leq \int_{0}^{\infty} \frac{1}{1+t^{2}} d t+\lim _{T \rightarrow \infty} \int_{0}^{T} \tilde{u}(t) d t=\frac{\pi}{2} .
\end{aligned}
$$

Therefore, $\Omega_{\lim }^{\frac{\pi}{2}} \neq \emptyset$ and assumption $\left(I_{\lim } 6\right)$ is satisfied. Finally, observe that

$$
\frac{\sin ^{2}(x(t)) u^{2}(t)}{1+t^{2}}+u(t) \geq u(t) \geq \widetilde{u}(t) \text { for a.e. } t \in[0, \infty[
$$

for any pair $(x, u) \in \Omega_{\lim } \supset \Omega_{\lim }^{\frac{\pi}{2}}$. This means that assumption $\left(I_{\lim } 7\right)$ is satisfied for $\lambda(t)=\widetilde{u}(t)$ (the function $\tilde{u}$ is locally summable on $[0, \infty[$ and $\left.\lim _{T \rightarrow \infty} \int_{0}^{T} \tilde{u}(t) d t=0\right)$. Theorem 7.9 implies that the control system $\left(\mathrm{P}^{2}\right)$ with the cost functional $\left(J_{\text {lim }}^{2}\right)$ has an almost strongly optimal solution $\left(x^{*}, u^{*}\right)$.

Observe that the pair $(\tilde{x}, \tilde{u})$ is not admissible for the control problem $\left(\mathrm{P}^{1}\right)$ with the cost functional $\left(J_{f}^{1}\right)$. 


\section{Optimality Principles}

Let us introduce the following definition; see [3].

Definition 8.1 A pair $\left(x^{*}, u^{*}\right)$ where $x^{*} \in A C_{\text {loc }}\left(\left[0, \infty\left[, \mathbb{R}^{n}\right)\right.\right.$ and $u^{*}:\left[0, \infty\left[\rightarrow \mathbb{R}^{m}\right.\right.$ is a measurable function is called finitely optimal iff it satisfies system $(\mathrm{P})$ on $[0, T]$; the integral $\int_{0}^{T} F\left(t, x^{*}(t), u^{*}(t)\right) d t$ is finite; and the inequality

$$
\int_{0}^{T} F\left(t, x^{*}(t), u^{*}(t)\right) d t \leq \int_{0}^{T} F(t, x(t), u(t)) d t
$$

holds true for each pair $(x, u):[0, T] \rightarrow \mathbb{R}^{n} \times \mathbb{R}^{m}$ where $x$ is an absolutely continuous function on $[0, T]$ and $u$ is a measurable function on $[0, T]$, such that they satisfy system $(\mathrm{P})$ on $[0, T]$ and the condition

$$
x(T)=x^{*}(T),
$$

and such that the function $F(\cdot, x(\cdot), u(\cdot))$ is integrable on $[0, T]$.

\subsection{Case of a (Proper) Lebesgue Integral}

The method of proving the next theorem is similar to that presented in [3, Theorem 2.2]. We give this proof here to complete the task.

Theorem 8.2 If a pair $\left(x^{*}, u^{*}\right) \in \Omega_{\int}$ is a classical optimal pair then it is finitely optimal.

Proof Assume, for contradiction, that a classical optimal pair $\left(x^{*}, u^{*}\right) \in \Omega \int$ is not finitely optimal. Then there exist some $T>0$ and a pair of functions $\left(x^{+}, u^{+}\right)$: $[0, T] \rightarrow \mathbb{R}^{n} \times \mathbb{R}^{m}$ where $x^{+}$is absolutely continuous on $[0, T]$ and $u^{+}$is measurable on $[0, T]$ such that they satisfy system $(\mathrm{P})$ on $[0, T]$, condition $(22)$, and the inequality

$$
\int_{0}^{T} F\left(t, x^{+}(t), u^{+}(t)\right) d t<\int_{0}^{T} F\left(t, x^{*}(t), u^{*}(t)\right) d t .
$$

In this case, there exists an $\varepsilon>0$ such that

$$
\int_{0}^{T} F\left(t, x^{+}(t), u^{+}(t)\right) d t<\int_{0}^{T} F\left(t, x^{*}(t), u^{*}(t)\right) d t-\varepsilon .
$$

Let $(\tilde{x}, \tilde{u}):\left[0, \infty\left[\rightarrow \mathbb{R}^{n} \times \mathbb{R}^{m}\right.\right.$ be defined by

$$
(\tilde{x}(t), \tilde{u}(t))= \begin{cases}\left(x^{+}(t), u^{+}(t)\right), & t \in[0, T], \\ \left(x^{*}(t), u^{*}(t)\right), & t \in] T, \infty[.\end{cases}
$$

First, we shall prove that $(\tilde{x}, \tilde{u}) \in \Omega_{f}$; see Definition 7.4. Obviously, $\tilde{x} \in$ $A C_{\text {loc }}\left(\left[0, \infty\left[, \mathbb{R}^{n}\right)\right.\right.$, the function $\tilde{u}$ is measurable, and the pair $(\tilde{x}, \tilde{u})$ satisfies system $(\mathrm{P})$ on $[0, \infty[$. Moreover, the function $F(\cdot, \tilde{x}(\cdot), \tilde{u}(\cdot))$ is integrable on $[0, \infty[$. 
Indeed, since the integral $\int_{0}^{T} F\left(t, x^{+}(t), u^{+}(t)\right) d t$ exists, therefore,

$$
\begin{aligned}
\int_{0}^{T} F\left(t, x^{+}(t), u^{+}(t)\right) d t= & \int_{0}^{T}\left[F\left(t, x^{+}(t), u^{+}(t)\right)\right]_{+} d t \\
& -\int_{0}^{T}\left[F\left(t, x^{+}(t), u^{+}(t)\right)\right]_{-} d t
\end{aligned}
$$

where $\left[F\left(\cdot, x^{+}(\cdot), u^{+}(\cdot)\right)\right]_{+}$and $\left[F\left(\cdot, x^{+}(\cdot), u^{+}(\cdot)\right)\right]_{-}$are the positive and negative parts of the function $F\left(\cdot, x^{+}(\cdot), u^{+}(\cdot)\right)$, and at least one of the integrals on the righthand side is finite; see the definition of integrability in Sect. 2. Assume that the integral

$$
\int_{0}^{T}\left[F\left(t, x^{+}(t), u^{+}(t)\right)\right]_{+} d t
$$

is finite. The pair $\left(x^{*}, u^{*}\right)$ is a classical optimal pair, hence

$$
\begin{aligned}
\int_{0}^{\infty} F\left(t, x^{*}(t), u^{*}(t)\right) d t= & \int_{0}^{\infty}\left[F\left(t, x^{*}(t), u^{*}(t)\right)\right]_{+} d t \\
& -\int_{0}^{\infty}\left[F\left(t, x^{*}(t), u^{*}(t)\right)\right]_{-} d t
\end{aligned}
$$

and both integrals on the right-hand side are finite, because $J_{\int}\left(z^{*}, u^{*}\right) \in \mathbb{R}$. Since

$$
[F(t, \tilde{x}(t), \tilde{u}(t))]_{+}= \begin{cases}{\left[F\left(t, x^{+}(t), u^{+}(t)\right)\right]_{+},} & t \in[0, T], \\ {\left[F\left(t, x^{*}(t), u^{*}(t)\right)\right]_{+},} & t \in] T, \infty[\end{cases}
$$

therefore,

$$
\begin{aligned}
\int_{0}^{\infty}[F(t, \tilde{x}(t), \tilde{u}(t))]_{+} d t= & \int_{0}^{T}\left[F\left(t, x^{+}(t), u^{+}(t)\right)\right]_{+} d t \\
& +\int_{T}^{\infty}\left[F\left(t, x^{*}(t), u^{*}(t)\right)\right]_{+} d t \in \mathbb{R}
\end{aligned}
$$

the second integral on the right-hand side is finite because the integral $\int_{0}^{\infty}\left[F\left(t, x^{*}(t), u^{*}(t)\right)\right]_{+} d t$ is finite. As a result, the integral $\int_{0}^{\infty} F(t, \tilde{x}(t)$, $\widetilde{u}(t)) d t$ exists. In a similar way, one can consider the case where the integral $\int_{0}^{T}\left[F\left(t, x^{+}(t), u^{+}(t)\right)\right]_{-} d t$ is finite. Hence, $(\tilde{x}, \tilde{u}) \in \Omega_{f}$. Since the pair $\left(x^{*}, u^{*}\right)$ is a classical optimal pair and (23) holds true, therefore,

$$
\begin{aligned}
\int_{0}^{\infty} F\left(t, x^{*}(t), u^{*}(t)\right) d t \\
\quad<\int_{0}^{\infty} F(t, \tilde{x}(t), \widetilde{u}(t)) d t+\frac{\varepsilon}{2}
\end{aligned}
$$




$$
\begin{aligned}
& =\int_{0}^{T} F\left(t, x^{+}(t), u^{+}(t)\right) d t+\int_{T}^{\infty} F\left(t, x^{*}(t), u^{*}(t)\right) d t+\frac{\varepsilon}{2} \\
& <\int_{0}^{\infty} F\left(t, x^{*}(t), u^{*}(t)\right) d t-\frac{\varepsilon}{2} .
\end{aligned}
$$

The contradiction completes the proof.

\subsection{Case of an Improper Lebesgue Integral}

Theorem 8.3 If a pair $\left(z^{*}, u^{*}\right) \in \Omega_{\lim }$ is almost strongly optimal, then it is finitely optimal.

Proof $\left({ }^{7}\right)$ Assume that the statement does not hold. Then there exists some $T>0$ and some pair of functions $\left(x^{+}, u^{+}\right):[0, T] \rightarrow \mathbb{R}^{n} \times \mathbb{R}^{m}$ where $x^{+}$is absolutely continuous on $[0, T]$ and $u^{+}$is measurable on $[0, T]$, such that they satisfy system (P) on $[0, T]$, the condition $x(T)=x^{+}(T)$, and the inequality

$$
\int_{0}^{T} F\left(t, x^{+}(t), u^{+}(t)\right) d t<\int_{0}^{T} F\left(t, z^{*}(t), u^{*}(t)\right) d t .
$$

Let $\varepsilon>0$ be such that

$$
\int_{0}^{T} F\left(t, x^{+}(t), u^{+}(t)\right) d t<\int_{0}^{T} F\left(t, x^{*}(t), u^{*}(t)\right) d t-\varepsilon .
$$

Consider the pair $(\tilde{x}, \tilde{u}):\left[0, \infty\left[\rightarrow \mathbb{R}^{n} \times \mathbb{R}^{m}\right.\right.$ given by (24). First, we shall prove that $(\tilde{x}, \tilde{u}) \in \Omega_{\mathrm{lim}}$. Obviously, $\tilde{x} \in A C_{\mathrm{loc}}\left(\left[0, \infty\left[, \mathbb{R}^{n}\right), \tilde{u}\right.\right.$ is a measurable function on $[0, \infty[$, and $(\tilde{x}, \tilde{u})$ satisfies system $(\mathrm{P})$ on $[0, \infty[$. Moreover, the function $F(\cdot, \tilde{x}(\cdot), \tilde{u}(\cdot))$ is locally integrable on $[0, \infty[$ and there exists a limit

$$
\lim _{T \rightarrow \infty} \int_{0}^{T} F(t, \tilde{x}(t), \tilde{u}(t)) d t,
$$

not necessarily finite. Indeed, since the integral $\int_{0}^{T} F\left(t, x^{+}(t), u^{+}(t)\right) d t$ exists, the following equality holds true:

$$
\begin{aligned}
& \int_{0}^{T} F\left(t, x^{+}(t), u^{+}(t)\right) d t \\
& \quad=\int_{0}^{T}\left[F\left(t, x^{+}(t), u^{+}(t)\right)\right]_{+} d t-\int_{0}^{T}\left[F\left(t, x^{+}(t), u^{+}(t)\right)\right]_{-} d t,
\end{aligned}
$$

where $\left[F\left(\cdot, x^{+}(\cdot), u^{+}(\cdot)\right)\right]_{+}$and $\left[F\left(\cdot, x^{+}(\cdot), u^{+}(\cdot)\right)\right]_{-}$are the positive and negative parts of the function $F\left(\cdot, x^{+}(\cdot), u^{+}(\cdot)\right)$ and at least one of the integrals on the righthand side is finite. Assume that the integral $\int_{0}^{T}\left[F\left(t, x^{+}(t), u^{+}(t)\right)\right]_{+} d t$ is finite.

\footnotetext{
${ }^{7}$ The proof of Theorem 8.3 is much the same as the proof of Theorem 8.2.
} 
Since the pair $\left(x^{*}, u^{*}\right)$ is almost strongly optimal, therefore,

$$
\begin{aligned}
& \int_{0}^{T_{1}} F\left(t, x^{*}(t), u^{*}(t)\right) d t \\
& \quad=\int_{0}^{T_{1}}\left[F\left(t, x^{+} *(t), u^{*}(t)\right)\right]_{+} d t-\int_{0}^{T_{1}}\left[F\left(t, x^{*}(t), u^{*}(t)\right)\right]_{-} d t
\end{aligned}
$$

for any $T_{1}>0$, and in this case the integrals on the right-hand side are finite. Consequently, for any $T_{1}>T$

$$
\begin{aligned}
& \int_{0}^{T_{1}} {[F(t, \tilde{x}(t), \tilde{u}(t))]_{+} d t } \\
& \quad=\int_{0}^{T}\left[F\left(t, x^{+}(t), u^{+}(t)\right)\right]_{+} d t+\int_{T}^{T_{1}}\left[F\left(t, x^{*}(t), u^{*}(t)\right)\right]_{+} d t \in \mathbb{R} .
\end{aligned}
$$

This means that the integral $\int_{0}^{T_{1}}[F(t, \widetilde{x}(t), \widetilde{u}(t))]_{+} d t$ is finite and, as a result, the integral $\int_{0}^{T_{1}} F(t, \tilde{x}(t), \tilde{u}(t)) d t$ exists for any $T_{1}>0$; the existence of such an integral for $T_{1} \leq T$ follows from the fact that $\left.(\tilde{x}, \tilde{u})\right|_{[0, T]}=\left(x^{+}, u^{+}\right)$. We shall prove that the limit

$$
\lim _{T \rightarrow \infty} \int_{0}^{T} F(t, \tilde{x}(t), \tilde{u}(t)) d t
$$

exists, not necessarily finite. Indeed, for $T_{1}>T$

$$
\begin{array}{rl}
\int_{0}^{T_{1}} & F(t, \tilde{x}(t), \tilde{u}(t)) d t \\
= & \int_{0}^{T} F\left(t, x^{+}(t), u^{+}(t)\right) d t+\int_{T}^{T_{1}} F\left(t, x^{*}(t), u^{*}(t)\right) d t \\
= & \int_{0}^{T} F\left(t, x^{+}(t), u^{+}(t)\right) d t+\int_{0}^{T_{1}} F\left(t, x^{*}(t), u^{*}(t)\right) d t \\
& -\int_{0}^{T} F\left(t, x^{*}(t), u^{*}(t)\right) d t .
\end{array}
$$

Since the pair $\left(x^{*}, u^{*}\right)$ is almost strongly optimal, there exists a finite limit

$$
\lim _{T_{1} \rightarrow \infty, T_{1}>T} \int_{0}^{T_{1}} F\left(t, x^{*}(t), u^{*}(t)\right) d t
$$

(the integral $\int_{0}^{T} F\left(t, x^{*}(t), u^{*}(t)\right) d t$ is finite, too) and, further, there exists a limit

$$
\lim _{T_{1} \rightarrow \infty} \int_{0}^{T_{1}} F(t, \tilde{x}(t), \tilde{u}(t)) d t .
$$


Hence, $(\tilde{x}, \tilde{u}) \in \Omega_{\lim }$. By the fact that the pair $\left(x^{*}, u^{*}\right)$ is almost strongly optimal, by (25), and (26),

$$
\begin{aligned}
& \lim _{T_{1} \rightarrow \infty} \int_{0}^{T_{1}} F\left(t, x^{*}(t), u^{*}(t)\right) d t \\
& <\lim _{T_{1} \rightarrow \infty} \int_{0}^{T_{1}} F(t, \tilde{x}(t), \tilde{u}(t)) d y+\frac{\varepsilon}{2} \\
& =\int_{0}^{T} F\left(t, x^{+}(t), u^{+}(t)\right) d t+\lim _{T_{1} \rightarrow \infty} \int_{0}^{T_{1}} F\left(t, x^{*}(t), u^{*}(t)\right) d t \\
& \quad-\int_{0}^{T} F\left(t, x^{*}(t), u^{*}(t)\right) d t+\frac{\varepsilon}{2} \\
& <\lim _{T_{1} \rightarrow \infty} \int_{0}^{T_{1}} F\left(t, x^{*}(t), u^{*}(t)\right) d t-\frac{\varepsilon}{2} .
\end{aligned}
$$

The contradiction completes the proof.

\section{Concluding Remarks}

We have considered an infinite-horizon optimal control problem with a cost functional given either by an integral over an unbounded interval (a Lebesgue integral) or by a limit of integrals (an improper Lebesgue integral). We have proposed natural definitions of optimality for these two models and stated some sufficient conditions for the existence of optimal solutions. The existence theorems are proven using the modified Lagrangian and some extensions of the lower closure theorem. The new definitions are compatible with the definitions for finite-horizon models (Theorem 8.2 and Theorem 8.3).

It seems reasonable to assume that similar tools can be used to determine sufficient conditions for the existence of optimal pairs for some models with cost functionals described by the lower and upper limits of Lebesgue integrals.

Acknowledgements The author is very grateful to Prof. Dariusz Idczak of the University of Łódź for his advice on this paper. The author would like to thank Dr. Marian Jakszto of the University of Łódź for pointing out the discussion of the improper Lebesgue integral in the literature.

Open Access This article is distributed under the terms of the Creative Commons Attribution License which permits any use, distribution, and reproduction in any medium, provided the original author(s) and the source are credited.

\section{References}

1. Sethi, S.P., Thompson, G.L.: Optimal Control Theory. Applications to Management Science and Economics. Springer, New York (2005) 
2. Carlson, D.: Some concepts of optimality for infinite horizon optimal control and their interrelationships. In: Modern Optimal Control. A Conference in Honor of Solomon Lefschetz and Joseph P. LaSalle, pp. 13-22. Dekker, New York (1989)

3. Carlson, D.A., Haurie, A.: Infinite Horizon Optimal Control. Theory and Applications. Springer, Berlin (1987)

4. Cesari, L.: Optimization-Theory and Applications. Problems with Ordinary Differential Equations. Springer, New York (1983)

5. Balder, E.J.: Lower closure problems with weak convergence conditions in a new perspective. SIAM J. Control Optim. 20(2), 198-210 (1982)

6. Carlson, D.A.: The controllability of infinite horizon optimal control problems. Nonlinear Anal. 11(4), 437-453 (1987)

7. Olech, C.: A characterization of $L_{1}$-weak lower semicontinuity of integral functionals. Bull. Acad. Pol. Sci., Sér. Sci. Math. Astron. Phys. 25(2), 135-142 (1977)

8. Łojasiewicz, S.: An Introduction to the Theory of Real Functions. Wiley, Chichester (1988)

9. Sikorski, R.: Funkcje Rzeczywiste. (Polish) [Real Functions], vol. I. Państwowe Wydawnictwo Naukowe (1958)

10. Sikorski, R.: Advanced Calculus. Functions of Several Variables. Państwowe Wydawnictwo Naukowe-Polish Scientific Publishers, (1969)

11. Lykina, V., Pickenhain, S., Wagner, M.: Different interpretations of the improper integral objective in an infinite horizon control problem. J. Math. Anal. Appl. 340(1), 498-510 (2008)

12. Rudin, W.: Functional Analysis. McGraw-Hill, New York (1991)

13. Rockafellar, R.T.: Integral functionals, normal integrands and measurable selections. In: Nonlinear Operators and the Calculus of Variations. Summer School Held at the Université Libre de Bruxelles, Brussels, 8-19 September 1975, pp. 157-207. Springer, Berlin (1976)

14. Balder, E.J.: An existence result for optimal economic growth problems. J. Math. Anal. Appl. 95(1), 195-213 (1983)

15. Giannessi, F.: Constrained Optimization and Image Space Analysis, vol. I. Springer, New York (2005) 